\title{
Desarrollo de un método de simulación física de zonas térmicamente afectadas en soldaduras de acero, para estudios de propagación de grietas por fatiga
}

\author{
Development of a physical simulation method of steel welds for \\ fatigue crack propagation studies, on heat affected zones
}

Desenvolvimento de um método de simulação física de zonas
termicamente afetadas em soldagens de aço, para estudos de
propagação de fissuras por fadiga

Fecha de recepción: 18 de enero de 2016

Fecha de aprobación: 22 de abril de 2016

\author{
Daniel Fernando Atehortua-López* \\ Ramiro Catacolí-Pereira* \\ Yesid Aguilar-Castro \\ Héctor Sánchez-Sthepa*** \\ Ilchat Sabirov
}

\section{Resumen}

Se presenta el desarrollo de un método de simulación física, a través del sistema Gleeble, de las zonas térmicamente afectadas de uniones soldadas de aceros estructurales (A283 Gr. C y A106 Gr. B), para el estudio de la propagación de grietas por fatiga. Para ello fue necesario utilizar probetas de $90 \mathrm{~mm}$ de largo, $49,5 \mathrm{~mm}$ de ancho y $7 \mathrm{~mm}$ de espesor, en una configuración que no se encuentra dentro de los estándares del sistema para este tipo de simulaciones. Con este método se reprodujeron satisfactoriamente, y por separado, en diferentes probetas del metal base correspondiente, la zona de grano fino, la zona de grano grueso y una zona de múltiples pasadas presentes en uniones soldadas fabricadas por FCAW. Las zonas simuladas fueron reproducibles, homogéneas, no presentaron defectos ni tensiones residuales y tuvieron un tamaño considerablemente grande, además que las características microestructurales (como el tamaño de grano y el porcentaje de fases) fueron muy similares a su contraparte real, lo que permitió estudiar por primera vez el efecto neto de la microestructura sobre el comportamiento a la fatiga

\footnotetext{
* Ph.D. Universidad del Valle (Cali - Valle del Cauca, Colombia); Universidad Carlos III (Leganés - Madrid, España). daniel.atehortua@correounivalle.edu.co.

** Universidad del Valle (Cali - Valle del Cauca, Colombia).

*** Ph.D. Universidad del Valle (Cali - Valle del Cauca, Colombia). yesid.aguilar@correounivalle.edu.co.

**** Ph.D. Universidad del Valle (Cali - Valle del Cauca, Colombia).

***** Ph.D. Instituto Madrileño de Estudios Avanzados de Materiales (Getafe - Madrid, España)
} 
Desarrollo de un método de simulación física de zonas térmicamente afectadas en soldaduras de acero, para estudios de propagación de grietas por fatiga

en estas zonas. De esta manera, el desarrollo realizado permitió aumentar considerablemente las aplicaciones y las ventajas que esta técnica puede tener, además de mejorar la comprensión del comportamiento a la fatiga de las soldaduras de acero.

Palabras Clave: propagación de grietas por fatiga, simulación física de la ZTA, uniones soldadas de aceros estructurales, variación microestructural en la ZTA.

\section{Abstract}

In order to study the fatigue crack propagation in the Heat Affected Zone (HAZ) of structural steel weld joints, test specimens of $90 \mathrm{~mm} \times 49,5 \mathrm{~mm} \times 7 \mathrm{~mm}$ in size were used to carry out the simulations, for which a special procedure was required to be developed in the Gleeble system. By doing so, the fine grain zone, the coarse grain zone and the multi-pass zone of a weld joint produced by FCAW were successfully reproduced separately on different samples of the corresponding base metal. The simulated zones were reproducible, homogeneous, defectfree, residual-stress-free and large enough; besides their microstructural characteristics (such as grain size and phase percentage) were very close to their real counterparts, which made it possible to study the actual effect of HAZ microstructure on the fatigue crack propagation rate in these zones for the first time. Therefore, the development enabled to significantly increase applications and the advantages of this technique, while allowed improving the understanding of fatigue behavior in steel weld joints.

Keywords: fatigue crack propagation, HAZ microstructural variation, HAZ physical simulation, structural steel welding.

\section{Resumo}

Neste trabalho se apresenta o desenvolvimento de um método de simulação física, através do sistema Gleeble, das zonas termicamente afetadas de uniões soldadas de aços estruturais (A283 Gr. C e A106 Gr. B), para o estudo da propagação de fissuras por fadiga. Para isso foi necessário utilizar provetas de $90 \mathrm{~mm}$ de comprimento, 49,5 mm de largura e $7 \mathrm{~mm}$ de espessura, em uma configuração que não se encontra dentro dos padrões do sistema para este tipo de simulações. Com este método se reproduziram satisfatoriamente, e por separado, em diferentes provetas do metal base correspondente, a zona de grão fino, a zona de grão grosso e uma zona de múltiplas passadas presentes em uniões soldadas fabricadas por FCAW. As zonas simuladas foram reproduzíveis, homogêneas, não apresentaram defeitos nem tensões residuais e tiveram um tamanho consideravelmente grande, além de que as características microestruturais (como o tamanho de grão e a porcentagem de fases) foram muito similares a sua contraparte real, o que permitiu estudar por primeira vez o efeito líquido da microestrutura sobre o comportamento à fadiga nestas zonas. Desta maneira, o desenvolvimento realizado permitiu aumentar consideravelmente as aplicações e as vantagens que esta técnica pode ter, além de melhorar a compreensão do comportamento à fadiga das soldas de aço.

Palavras Chave: simulação física da ZTA, variação microestrutural na ZTA, uniões soldadas de aços estruturais, propagação de fissuras por fadiga.

Cómo citar este artículo:

[1] D. F. Atehortua-López, R. Catacolí-Pereira, Y.Aguilar-Castro, H. Sánchez-Sthepa and I. Sabirov, “Desarrollo de un método de simulación física de zonas térmicamente afectadas en soldaduras de acero, para estudios de propagación de grietas por fatiga", Fac. Ing., vol. 25 (42), pp. 33-54 may.-ago. 2016. 


\section{INTRODUCCIÓN}

Es bien sabido que las fallas por fatiga que se presentan en las estructuras soldadas, como puentes, barcos, plataformas marítimas, recipientes a presión y edificios, ocurren, predominantemente, en las uniones soldadas, a pesar de que dichas estructuras hayan sido diseñadas, construidas e inspeccionadas de acuerdo con los códigos de construcción soldada correspondientes [1]. La evidencia recogida de estas fallas muestra que la mayoría de las grietas por fatiga en uniones soldadas inician en el pie de la soldadura o en la terminación del cordón y se propagan a través de la zona térmicamente afectada (ZTA), dependiendo de la geometría de la junta $[1,2]$.

A pesar de la importancia que tiene la ZTA en la propagación de grietas por fatiga en uniones soldadas de aceros, los códigos de construcción soldada desprecian el efecto de la variación microestructural que se presenta en dicha zona sobre el fenómeno de propagación de la grieta, y las propiedades del material en la determinación de la vida útil del componente soldado toman un papel secundario [2]. El motivo de tal simplificación está relacionado con el hecho de que la velocidad de propagación de grietas por fatiga es independiente de la composición química, la microestructura y las propiedades mecánicas del acero, según lo que se ha afirmado hasta ahora en la literatura $[1,3,4]$. No obstante, tal afirmación resulta paradójica si se tiene en cuenta la vulnerabilidad que representan los distintos factores adversos que se conjugan en la ZTA [2], lo cual ha suscitado gran interés entre la comunidad científica a través del tiempo.

A pesar de ello, el estudio de la ZTA se ha limitado hasta el momento a realizar caracterizaciones microestructurales y mecánicas, principalmente a través de mediciones de microdureza, debido al tamaño reducido que presentan estas zonas en la unión soldada. Por tal motivo, la simulación física de la ZTA por medio del sistema Gleeble ha jugado un papel importante. Los beneficios de la utilización de este sistema han quedado demostrados ampliamente en numerosas investigaciones [5-10]. No obstante, existen también algunas desventajas; una de las más importantes radica en el tamaño reducido de las probetas estándar que se utilizan para llevar a cabo dichas simulaciones (barras de sección transversal circular de $6 \mathrm{~mm}$ de diámetro y $76 \mathrm{~mm}$ de largo o barras de sección transversal cuadrada de $10 \mathrm{~mm} \times$ $10 \mathrm{~mm}$ y $76 \mathrm{~mm}$ de largo), lo cual limita el tipo de ensayos posteriores que se pueden realizar con estas probetas simuladas. Por consiguiente, para poder llevar a cabo pruebas de propagación de grietas por fatiga bajo los requerimientos de la norma de ensayo correspondiente, como la ASTM E647-13 $\mathrm{a}^{\mathrm{e}}$, en muestras representativas de la ZTA de una soldadura, obtenidas a través de simulación física por medio del sistema Gleeble, se deben utilizar probetas con geometría diferente al estándar en la simulación física, de modo que se posibilite la mecanización posterior de probetas tipo CT (compact tension) u otras permitidas por la norma. Es por esta razón que se requiere el desarrollo de un procedimiento especial en el sistema Gleeble que permita la reproducción de las características microestructurales de la ZTA utilizando probetas con la geometría apropiada. De esta forma, es posible estudiar si realmente existe un efecto de la variación microestructural que se presenta en la ZTA sobre la propagación de grietas por fatiga en uniones soldadas de aceros.

Ese fue el objetivo del trabajo que se describe a continuación. Para ello, se utilizaron probetas tipo placa de $90 \mathrm{~mm}$ de largo, 49,5 $\mathrm{mm}$ de ancho y 7 mm de espesor en la simulación de las ZTA más representativas de uniones soldadas de acero A283 Gr. Cy A106 Gr. B, como son la zona de grano fino, la zona de grano grueso y una zona de múltiples pasadas [2]. Estas zonas se presentan típicamente como resultado de la fabricación de la junta a través del proceso de arco con núcleo fundente ( $F C A W$, por sus siglas en inglés). El tamaño de estas probetas resultó de la necesidad de mecanizar posteriormente la geometría que se detalla en la Figura 1, dimensionada de acuerdo con los lineamientos de la norma de ensayo ASTM E647 - $13 \mathrm{a}^{\mathrm{e} 1}$. 


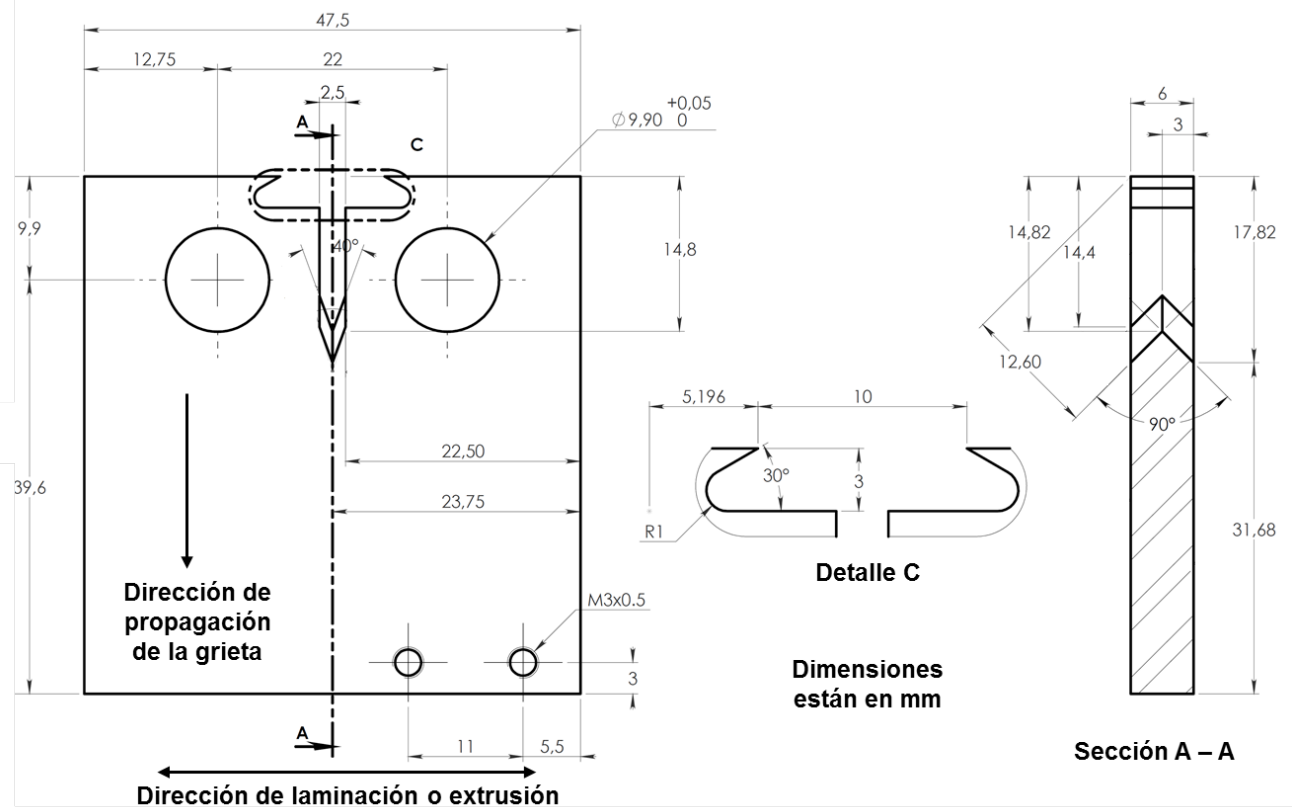

Fig. 1. Geometría de la probeta tipo CT utilizada en los estudios de propagación de grietas por fatiga.

\section{Procedimiento experimental}

\section{A. Materiales}

Para fabricar las uniones soldadas se utilizaron placas de acero A283 Gr. C de $250 \mathrm{~mm}$ x $500 \mathrm{~mm}$ y $12,7 \mathrm{~mm}$ de espesor y tubería sin costura de $250 \mathrm{~mm}$ de largo con diámetro nominal $150 \mathrm{~mm}$ y Schedule 160 de acero A106 Gr. B. De la misma fuente se mecanizaron las probetas que se utilizaron para llevar a cabo la simulación física. La composición química de los metales base, medida a través de espectrometría de masas, se encuentra en la Tabla 1.

TABla 1

COMPOSICIÓN QUÍMICA DE LOS MATERIALES UTILIZADOS

\begin{tabular}{|c|c|c|c|c|c|c|c|c|c|c|c|c|}
\hline \multirow{2}{*}{ Acero } & \multirow{2}{*}{ Grado } & \multicolumn{10}{|c|}{ Composición Nominal (\% peso) } \\
\cline { 3 - 26 } & & $\mathbf{C}$ & $\mathbf{M n}$ & $\mathbf{S i}$ & $\mathbf{S}$ & $\mathbf{P}$ & $\mathbf{N i}$ & $\mathbf{C r}$ & $\mathbf{C u}$ & $\mathbf{M o}$ & $\mathbf{V}$ & Fe \\
\hline A283 & C & 0,175 & 0,721 & 0,233 & 0,011 & 0,001 & 0,018 & 0,020 & 0,015 & 0,008 & 0,001 & Balance \\
\hline A106 & B & 0,200 & 0,500 & 0,268 & 0,004 & 0,002 & 0,047 & 0,092 & 0,120 & 0,016 & 0,002 & Balance \\
\hline
\end{tabular}

\section{B. Procedimiento de soldadura}

Las uniones soldadas por FCAW se fabricaron de acuerdo con las especificaciones del procedimiento de soldadura establecidas para cada metal base. Estas especificaciones fueron diseñadas y evaluadas siguiendo los lineamientos de los códigos de construcción de estructuras soldadas, de la Sociedad Americana de Soldadura ( $A W S$, por sus siglas en inglés), y los códigos de Calderas y Recipientes a Presión, de la Sociedad Americana de Ingenieros Mecánicos ( $A S M E$, por sus siglas en inglés). Las características completas de los procedimientos de soldadura empleados se pueden verificar en [2]. El proceso de soldadura por FCAW es ampliamente utilizado en la industria para la construcción por soldadura de estructuras sometidas a condiciones de fatiga, como los recipientes a presión y las tuberías. 


\section{Caracterización microestructural}

En primer lugar, para identificar y seleccionar las zonas objeto de estudio se realizó un macroataque a la sección transversal de las uniones soldadas con una solución de $15 \mathrm{ml}$ de ácido nítrico, $5 \mathrm{ml}$ de etanol y $85 \mathrm{ml}$ de agua. Las zonas identificadas de esta forma fueron posteriormente caracterizadas a través de Microscopía Óptica (MO) y Microscopía Electrónica de Barrido (MEB). Para ello, las superficies con acabado tipo espejo fueron atacadas inicialmente con una solución de picral al 4\%; este ataque sirvió para cuantificar el porcentaje de ferrita total presente en cada microestructura, con ayuda de un analizador de imágenes y las micrografías tomadas a través de MO. Luego de ello, y sin remover la microestructura revelada con picral, se atacó con nital al $2 \%$ para definir los bordes de grano y determinar el tamaño de grano en el metal base, en la zona de grano fino y en la zona de múltiples pasadas, de acuerdo con la norma ASTM E112-10. Por las morfologías tan irregulares de los granos presentes en la zona de grano grueso, no se llevó a cabo la medición del tamaño de grano en esta zona. El mismo procedimiento se utilizó para estudiar la microestructura de las probetas simuladas sobre el plano perpendicular a la trayectoria de propagación de la grieta. Las probetas tipo placa se mecanizaron de manera que este plano coincidió con el plano de la sección transversal estudiada en las uniones soldadas. El tamaño de grano y el porcentaje de ferrita total (determinado a través de la suma de los diferentes tipos de ferrita presentes en la microestructura) se utilizaron como parámetros cuantitativos para evaluar el grado de éxito de la simulación, junto con parámetros cualitativos, como la identificación de las fases observadas en la microestructura.

\section{Simulación física de las ZTA objeto de estudio}

Para la simulación de las zonas de grano fino y las zonas de grano grueso que se produjeron en las uniones soldadas fabricadas por FCAW se tomó como referencia la ZTA de los últimos pases o cordones de soldadura, debido a que la microestructura desarrollada reprodujo características de un procedimiento de pasada simple, al no encontrarse alterada por la aplicación de cordones de soldadura posteriores. Por su parte, la referencia para la simulación de la zona de múltiples pasadas correspondió a una zona de la junta afectada por la aplicación de dos pases de soldadura consecutivos.
Las simulaciones también se llevaron a cabo en especímenes de sección transversal cuadrada de 11 $\mathrm{mm}$ de ancho x $11 \mathrm{~mm}$ de espesor x $90 \mathrm{~mm}$ de largo, a partir de las cuales se mecanizaron probetas para ensayos de tensión.

La simulación física de las ZTA se realizó en un sistema Gleeble 3800. Se permitió la libre expansión y contracción térmica de la probeta a lo largo de su eje longitudinal durante las corridas, manteniendo en cero el nivel de fuerza medido por el sistema; de esta forma se evitó la aparición de esfuerzos residuales. Los ciclos térmicos utilizados en las simulaciones se calcularon a través del método $\mathrm{F}(\mathrm{s}, \mathrm{d})$, que permite reproducir la variación de temperatura en el tiempo de forma más precisa, en comparación con otros métodos de cálculo [11]. La curva de temperatura en función del tiempo, calculada de esta forma, quedó determinada principalmente por el aporte de calor y la temperatura máxima alcanzada en la zona; sin embargo, la etapa de calentamiento del ciclo térmico fue alterada, para evitar problemas de funcionamiento del sistema, como se explicará más adelante.

\section{E. Caracterización mecánica}

Los ensayos de tensión se llevaron a cabo en una máquina Tinius Olsen H50KS, de acuerdo con la norma de ensayo ASTM E8/E8M-13a, a temperatura ambiente. La velocidad de desplazamiento del cabezal fue de $3 \mathrm{~mm} / \mathrm{min}$. Las probetas utilizadas en el ensayo, cuya geometría se encuentra definida en la norma de ensayo mencionada, fueron mecanizadas a partir de las probetas de sección transversal cuadrada utilizadas en la simulación. La longitud calibrada fue de $16 \mathrm{~mm}$. A manera de comparación, los ensayos se realizaron también en probetas del metal base correspondiente.

Las mediciones de microdureza Vickers fueron tomadas en un equipo Wilson Instruments 401MVD, de acuerdo con la norma de ensayo ASTM E384-11. La carga utilizada fue $100 \mathrm{~g}$, y el tiempo de sostenimiento fue igual a $15 \mathrm{~s}$. Con respecto a las uniones soldadas, se realizaron barridos de microdureza sobre la sección transversal de cada unión orientados paralelamente a la superficie de las placas, de manera que cada indentación quedó equidistantemente espaciada 0,125 $\mathrm{mm}$ de la siguiente. Para obtener una estadística suficiente se realizaron cuatro barridos sobre el cordón de pasada simple escogido como referencia y el mismo número sobre el cordón de pasadas múltiples, 
Desarrollo de un método de simulación física de zonas térmicamente afectadas en soldaduras de acero, para estudios de propagación de grietas por fatiga

y se identificó a qué zona de la junta correspondió cada indentación, gracias a un ataque posterior con la solución de nital al $2 \%$. Por su parte, se realizaron barridos de microdureza en las probetas simuladas a lo largo y ancho de su sección transversal, con indentaciones equidistantemente espaciadas $0,5 \mathrm{~mm}$ de la siguiente, dentro de la sección procesada.

Finalmente, con el objeto de demostrar las ventajas que representa la simulación física de la ZTA de uniones soldadas de aceros a través del método desarrollado, al realizar ensayos de propagación de grietas por fatiga, se llevaron a cabo pruebas dinámicas bajo la norma ASTM E647-13a ${ }^{\text {el }}$ en una máquina Hung-Ta HT 9711. A partir de las placas utilizadas en la simulación física se mecanizaron probetas tipo CT, de acuerdo con la geometría que se muestra en la Fig. 1. Los ensayos se realizaron con amplitud de carga constante, relación entre carga mínima y carga máxima $(\mathrm{R})$ igual a 0,05 y frecuencia entre $30 \mathrm{~Hz}$ y $40 \mathrm{~Hz}$, a temperatura ambiente. También se ensayaron probetas del metal base correspondiente.

\section{RESUltados Y DiscuSióN}

\section{A. Características generales de las uniones soldadas}

Los resultados del macroataque se visualizan en la Fig. 2; esto permitió escoger las zonas que fueron simuladas a través del sistema Gleeble. En la Tabla 2 se resumen los parámetros más importantes del proceso de soldadura correspondientes a los cordones que produjeron las zonas seleccionadas. El aporte de calor se calculó tomando una eficiencia típica del proceso de soldadura por FCAW igual a 0,8 [12].

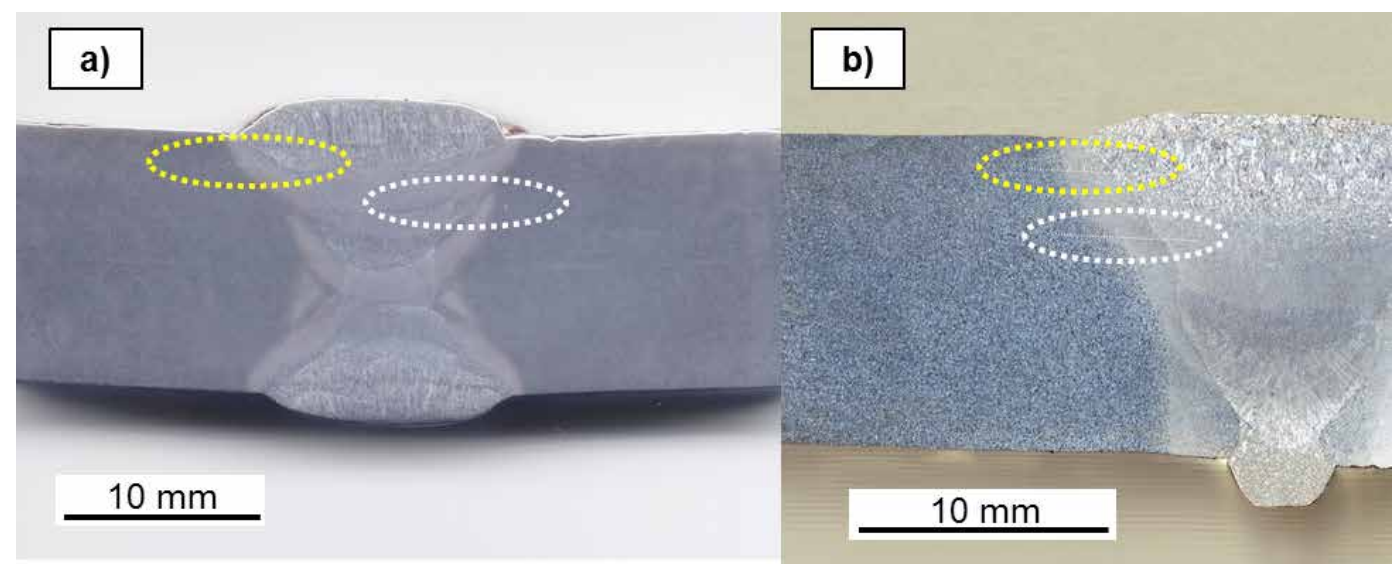

Fig. 2. a) Macroataque de la sección transversal de la unión soldada de acero A283 Gr. C; b) Macroataque de la sección transversal de la unión soldada de acero A106 Gr. B. Las regiones encerradas por una elipse amarilla correspondieron a las zonas de pasada simple seleccionadas, mientras que las encerradas por una elipse blanca representaron las zonas de múltiples pasadas que fueron objeto de estudio. 


\section{TABLA 2}

PARÁMETROS PRINCIPALES DEL PROCESO DE SOLDADURA UTILIZADOS EN LA APLICACIÓN DE LOS CORDONES QUE ORIGINARON LAS ZONAS OBJETO DE ESTUDIO. LOS CORDONES IDENTIFICADOS COMO PASADA SIMPLE ORIGINARON LAS ZONAS DE GRANO FINO Y GRANO GRUESO SELECCIONADAS

\begin{tabular}{|c|c|c|c|c|c|c|}
\hline Acero & Cordón & Amperaje (A) & Voltaje (V) & $\begin{array}{l}\text { Velocidad de } \\
\text { avance }(\mathrm{cm} / \mathrm{s})\end{array}$ & $\begin{array}{c}\text { Aporte de } \\
\text { Calor }(\mathrm{kJ} / \mathrm{cm})\end{array}$ & Observaciones \\
\hline A106 Gr. B & 3 & 165 & 24,7 & 0,24 & 13,6 & Múltiples pasadas \\
\hline A106 Gr. B & 4 & 165 & 24,8 & 0,20 & 16,4 & Pasada simple* \\
\hline A283 Gr. C & 4 & 205 & 28 & 0,41 & 11,2 & Múltiples pasadas \\
\hline A283 Gr. C & 5 & 205 & 27 & 0,50 & 8,9 & Múltiples pasadas \\
\hline A283 Gr. C & 6 & 205 & 28 & 0,39 & 11,8 & Pasada simple \\
\hline
\end{tabular}

* La aplicación de este cordón originó también la ZTA de múltiples pasadas

A manera de ilustración, en la Fig. 3 se muestran los resultados de uno de los barridos de microdureza realizados en las ZTA de pasada simple escogidas. Es evidente la variabilidad de las mediciones de dureza tomadas dentro de cada zona identificada. En esta figura se puede apreciar también la variación microestructural que se presenta en la ZTA, desde el metal base hacia la zona fundida, como resultado de dos factores principalmente: la temperatura máxima que alcanza cada zona $\left(\mathrm{T}_{\max }\right)$ y la velocidad de enfriamiento posterior. A medida que el metal se encuentra más cerca de la fuente de calor, la temperatura máxima alcanzada se incrementa. $\mathrm{Si}$ esta temperatura es lo suficientemente alta como para transformar la microestructura del acero (por encima de la línea A1 del diagrama de fases Fe - C), se genera un refinamiento parcial de grano que se incrementa de forma progresiva hasta que el metal se transforma completamente en austenita durante el calentamiento (temperatura ligeramente superior a la línea A3 del diagrama de fases $\mathrm{Fe}$ - C). A partir de este punto, conocido como la zona de grano fino, el aumento adicional de temperatura lleva a un incremento gradual del tamaño de grano austenítico, lo que ocasiona una reducción en el contenido de ferrita total producido durante el enfriamiento posterior por la disminución del número de sitios de nucleación de ferrita. A esta zona se le conoce como la zona de grano grueso. De esta manera se explica el aumento de dureza que se presenta desde el metal base hacia la zona fundida. 
Desarrollo de un método de simulación física de zonas térmicamente afectadas en soldaduras de acero, para estudios de propagación de grietas por fatiga
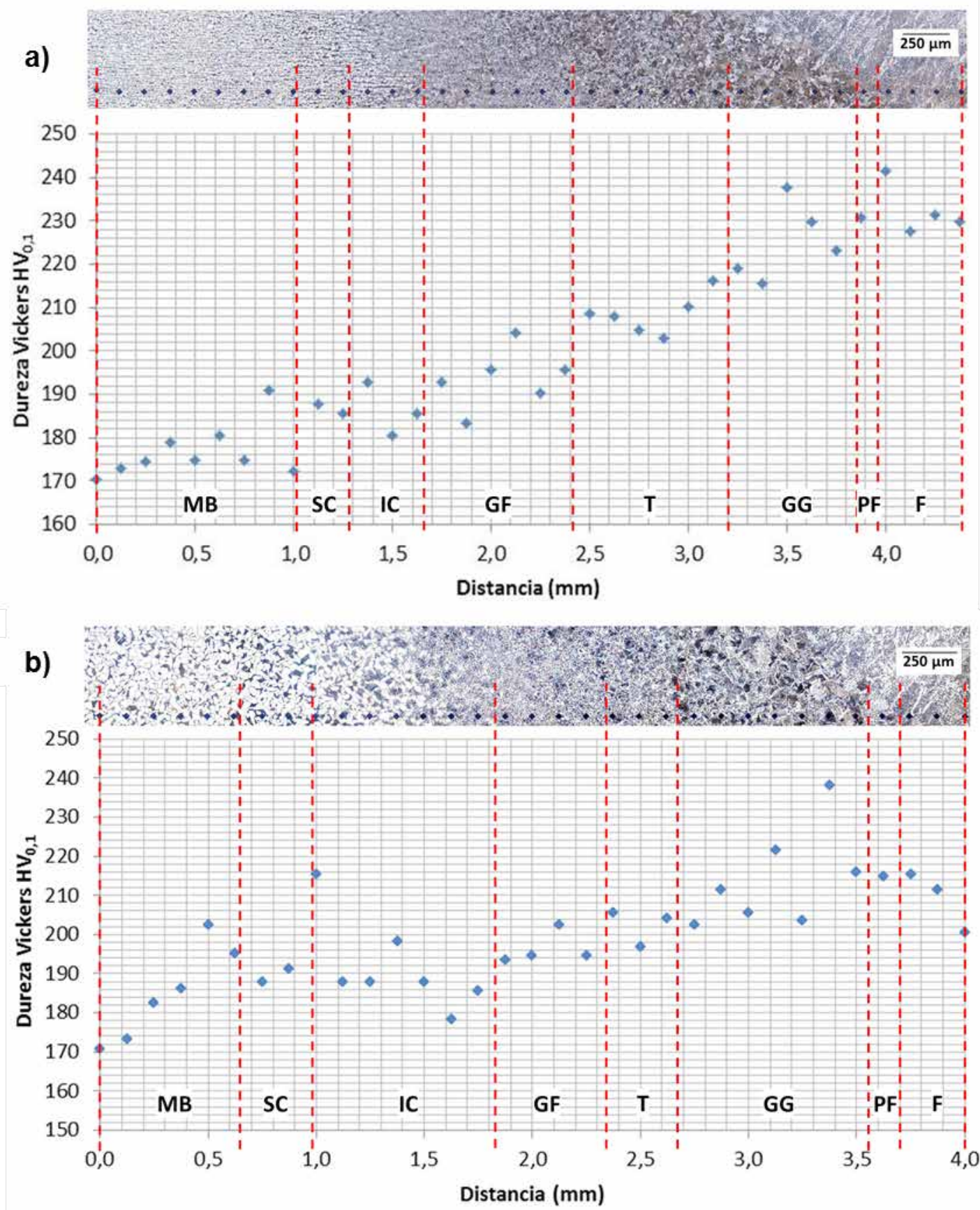

Fig. 3. a) Perfil de microdureza y la correspondiente microestructura a lo ancho de la ZTA del cordón 6 (pasada simple) de la unión soldada de acero A283 Gr. C; b) Perfil de microdureza y la correspondiente microestructura a lo ancho de la ZTA del cordón 4 (pasada simple) de la unión soldada de acero A106 Gr. B. MB = Metal base, SC = Zona subcrítica $\left(\mathrm{T}_{\max }<\mathrm{A} 1\right)$, $\mathrm{IC}=$ Zona intercrítica $\left(\mathrm{A} 1<\mathrm{T}_{\max }<\mathrm{A} 3\right), \mathrm{GF}=$ Zona de grano fino $\left(\mathrm{T}_{\max }>\mathrm{A} 3\right), \mathrm{T}=$ Zona de transición, $\mathrm{GG}_{\max }=$ Zona de grano grueso, $\mathrm{PF}=$ Zona parcialmente fundida, $\mathrm{F}=$ Zona fundida. Al hace referencia a la temperatura de transformación perlita-austenita. A3 hace referencia a la temperatura máxima de transformación ferrita-austenita para la aleación. 
En el caso de las zonas de múltiples pasadas, el calor transferido por la aplicación de un nuevo cordón de soldadura alcanzó a afectar una zona de grano grueso originada previamente (las demás zonas permanecieron sin alteraciones apreciables); sin embargo, por estar relativamente alejada de la zona fundida del cordón de soldadura posterior, la temperatura máxima alcanzada en esta zona solamente generó la homogeneización de la microestructura y el refinamiento del grano.

\section{B. Desarrollo del método de simulación física}

1) Descripción general: El sistema Gleeble 3800 es un equipo que permite variar la temperatura de una probeta metálica a una velocidad máxima de calentamiento o enfriamiento de $10.000{ }^{\circ} \mathrm{C} / \mathrm{s}$, suficiente para replicar el ciclo térmico al cual se somete el metal en la ZTA durante un procedimiento de soldadura por arco eléctrico. Para que tales velocidades puedan ser obtenidas, la probeta metálica debe ser lo suficientemente pequeña, lo que explica los tamaños de probeta estándar utilizados en este tipo de simulaciones. El calentamiento del metal se produce a través del efecto Joule, mientras que el enfriamiento se realiza principalmente por conducción de calor hacia las mordazas continuamente refrigeradas por agua (Fig. 4a). El calor se transfiere también por radiación y puede contribuir significativamente al proceso de enfriamiento a temperaturas elevadas. La temperatura de la probeta en función del tiempo, medida a través de termocuplas adheridas a la superficie de la muestra por medio de soldadura por resistencia (Fig. 4b), es el resultado de la competencia entre estos dos procesos mencionados. El sistema controla la cantidad de corriente eléctrica que fluye a través de la probeta para que alcance la temperatura que indica el programa de ensayo en determinado momento.

Debido a que la geometría propuesta lleva a un incremento del área de la sección transversal superior a 12 veces en comparación con las probetas estándar, mientras que el volumen de material por procesar se incrementa más de 60 veces, reproducir satisfactoriamente las microestructuras presentes en la ZTA de las uniones soldadas con estas nuevas probetas implica dificultades apreciables. Varios aspectos se tuvieron en cuenta al respecto:

- La demanda de corriente eléctrica aumenta con esta nueva configuración, que puede fácilmente superar la capacidad máxima del sistema. Por lo tanto, las velocidades de calentamiento se debieron restringir para evitar fallos durante la prueba.

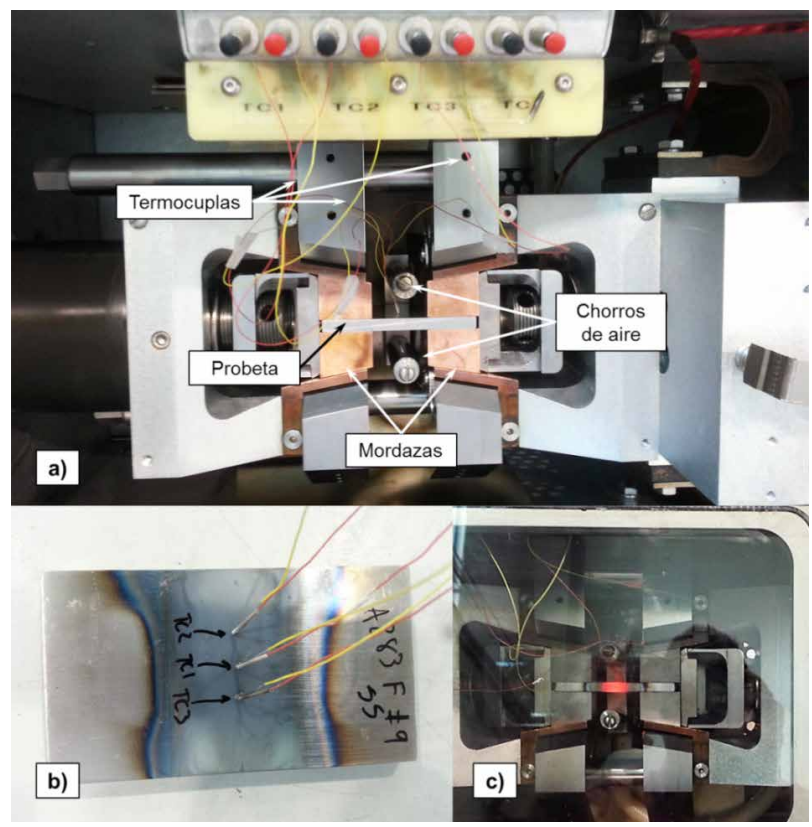

Fig. 4. a) Montaje experimental del procedimiento de simulación propuesto en el sistema Gleeble; b) Detalle de la probeta, se aprecia la ubicación de las diferentes termocuplas en el montaje. La aparición de diferentes

productos de corrosión (coloración distinta en la superficie), es un indicador del gradiente de temperatura que se presenta a lo largo del eje longitudinal; c) Prueba en proceso, se visualiza el gradiente de temperatura que se produce en la probeta.

- La cantidad de calor que se debe extraer de la probeta durante el enfriamiento es también muy elevada, y las mordazas no alcanzan a proporcionar la capacidad necesaria. Por lo tanto, se tuvo que mejorar la capacidad de extracción de calor con la aplicación de chorros de aire en ambos lados de la probeta, uniformemente distribuidos a lo ancho de esta (Fig. 4a). En este sentido, la presión de aire se utilizó como parámetro de control de la intensidad del enfriamiento.

- Se monitoreo la temperatura de la probeta en hasta tres puntos diferentes uniformemente espaciados a lo largo de la trayectoria de la grieta (Fig. 4b), uno de los cuales correspondió al control de temperatura (termocupla central). La caracterización microestructural descrita en II-C se llevó a cabo sobre los planos que contienen dichos puntos de monitoreo de temperatura. 
- Debido a la naturaleza del ensayo, es inevitable que se generen gradientes de temperatura sobre el eje longitudinal de la probeta, puesto que la temperatura es mínima en la región de contacto con las mordazas, y es máxima en el centro (Fig. 4c). Lo anterior significa que la microestructura de interés solo se puede reproducir satisfactoriamente en la sección central. El tamaño de esta zona procesada depende de varios parámetros del ensayo, entre ellos la separación de las mordazas, la temperatura máxima del ensayo y el material de las mordazas. En consecuencia, se garantizó una sección central simulada y homogénea suficientemente grande que posibilite la propagación de la grieta por fatiga dentro de la microestructura de interés
(Fig. 5). Para ello, la entalla de la probeta tipo CT (Fig. 1) se debió mecanizar en el centro de esta región procesada. El tamaño de esta sección simulada fue aproximadamente igual a $7 \mathrm{~mm}$ para la zona de grano fino, $12 \mathrm{~mm}$ para la zona de múltiples pasadas y $17 \mathrm{~mm}$ para la zona de grano grueso. Teniendo en cuenta que el ancho de toda la ZTA en las uniones soldadas reales fue inferior a $3 \mathrm{~mm}$ (Fig. 2 y Fig. 3), las probetas simuladas proporcionaron una sección significativamente más grande que representa una ventaja fundamental, pues permitió llevar a cabo pruebas de propagación de grietas por fatiga en este tipo de microestructuras.

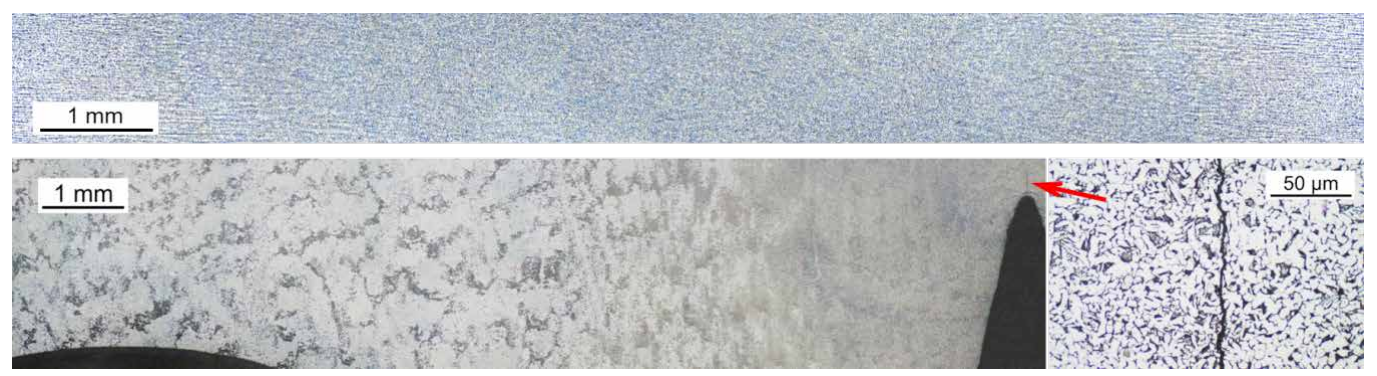

Fig. 5. Homogeneidad de la microestructura resultante en la sección simulada de algunas probetas. Se aprecia también el tamaño de esta sección. Arriba: zona de grano fino simulada para la soldadura de acero A283 Gr. C (probeta A283FS5). Abajo: probeta tipo CT para prueba de propagación de grietas por fatiga de la zona de grano fino simulada para la soldadura de acero A283 Gr. C (probeta A283-GS5); la grieta se propaga a lo largo de la microestructura simulada.

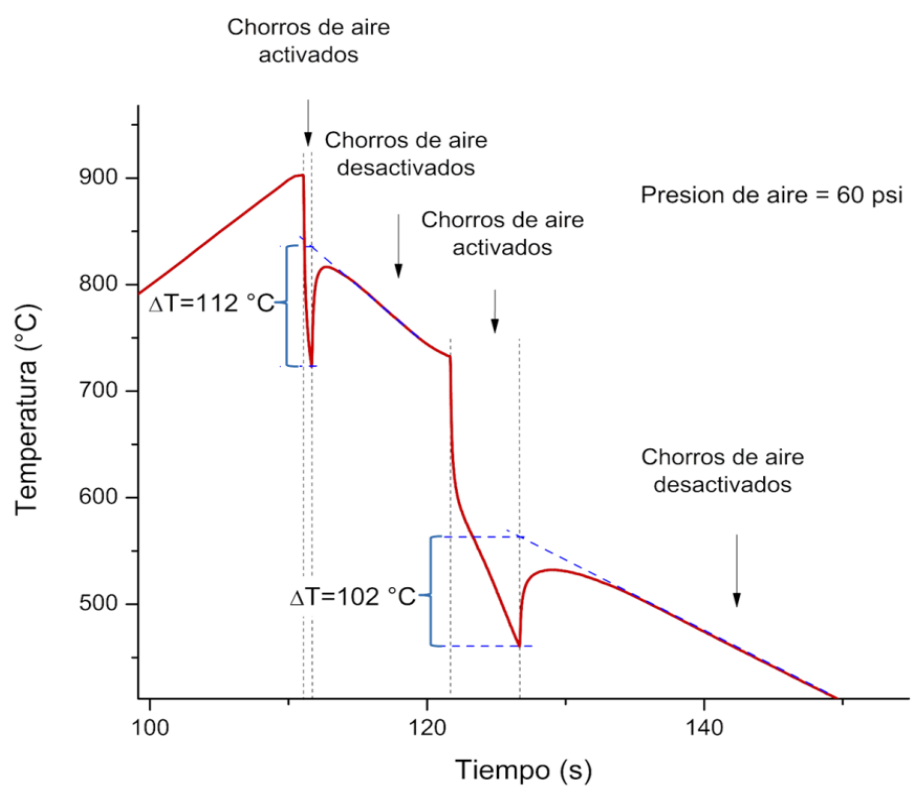

Fig. 6. El enfriamiento por chorros de aire produce un descenso artificial de la temperatura de la probeta, el cual se debe compensar en el programa de simulación. La diferencia de temperatura entre la superficie y el resto de la probeta depende de la presión de aire. 
- Como consecuencia de la utilización de chorros de aire durante el enfriamiento, la superficie de la probeta presentó una temperatura inferior al resto del material, lo cual se puede apreciar cualitativamente a través de la Fig. 6. Esta curva se obtuvo, en particular, al activar y desactivar los chorros de aire durante el enfriamiento sin la aplicación de corriente eléctrica. Debido a que la convección forzada contribuyó significativamente a la extracción de calor (mecanismo de transferencia superficial) y a que la temperatura se monitoreó precisamente en la superficie, se tuvo que modificar el programa de simulación para compensar la disminución en la temperatura medida. Con esta corrección, el efecto de la temperatura superficial inferior no ocasionó problemas de homogeneidad en la microestructura simulada, como se puede apreciar en la Fig. 7.
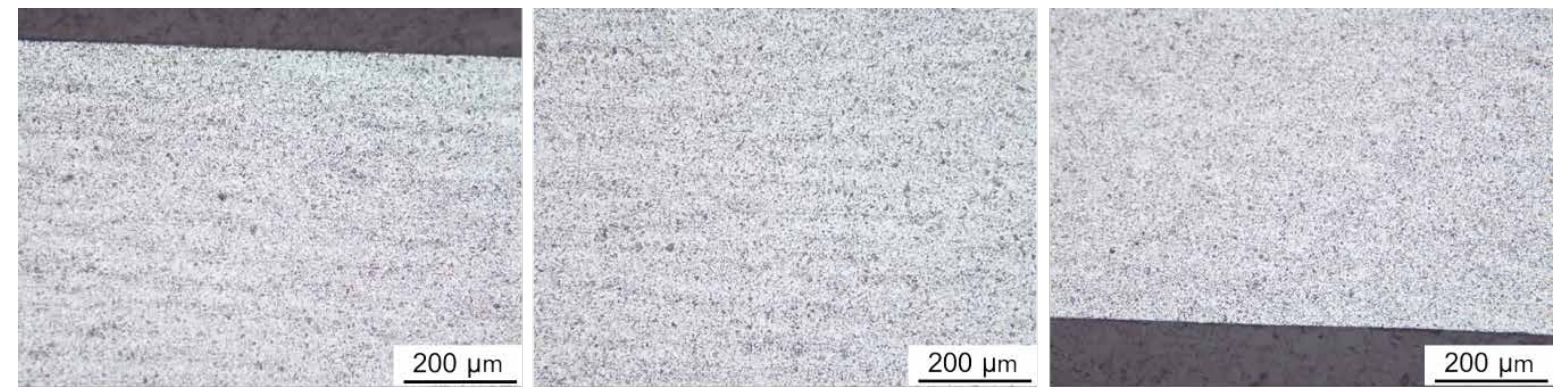

Fig. 7. El efecto de la temperatura superficial inferior no implicó una variación microestructural entre las superficies de la probeta (micrografías izquierda y derecha) y su centro (micrografía central). Probeta de grano fino (A283-FS5) simulada para la unión soldada de acero A283 Gr. C.

Para llevar a cabo las simulaciones con el método propuesto se utilizaron mordazas de cobre diferentes a las establecidas para este tipo de prueba. Estas mordazas son utilizadas normalmente para la simulación de procesos de recocido en probetas planas.

\section{2) Características de los ciclos térmicos utilizados} en las simulaciones: En todas las pruebas, la etapa de calentamiento se caracterizó por tener una velocidad constante limitada a $10{ }^{\circ} \mathrm{C} / \mathrm{s}$, por las razones expuestas previamente. De esta forma, las velocidades de calentamiento utilizadas fueron sensiblemente inferiores a las que se registran o calculan [por ejemplo, a través del método $\mathrm{F}(\mathrm{s}, \mathrm{d})$ ] durante la aplicación de un cordón de soldadura por arco eléctrico. Por lo general, estas velocidades pueden ser superiores a $500{ }^{\circ} \mathrm{C} / \mathrm{s}$, dependiendo del aporte de calor y la distancia de la zona a la fuente de calor. Sin embargo, debido a la composición química de los aceros utilizados en el estudio, esto no representó un problema que pudiera alterar la microestructura o las propiedades, puesto que no existen compuestos intermetálicos o precipitados diferentes al carburo de hierro en la microestructura original que pudieran solubilizarse en la matriz durante el calentamiento.
Como resultado del análisis de la ZTA en las uniones soldadas, se determinó la temperatura máxima para la simulación de las zonas de grano fino en $900{ }^{\circ} \mathrm{C}$ (temperatura ligeramente superior a la línea $\mathrm{A} 3$ del diagrama de fases $\mathrm{Fe}$ - $\mathrm{C}$ para la composición química de los aceros utilizados), mientras que la temperatura máxima para la simulación de las zonas de grano grueso fue $1200{ }^{\circ} \mathrm{C}$. Por su parte, la simulación de las zonas de múltiples pasadas se llevó a cabo con una primera corrida de zona de grano grueso (temperatura máxima de $1200{ }^{\circ} \mathrm{C}$ ), seguida por una corrida de zona de grano fino (temperatura máxima de $900{ }^{\circ} \mathrm{C}$ ). En todos los casos, el tiempo de sostenimiento a la temperatura máxima fue $1 \mathrm{~s}$.

La etapa de enfriamiento correspondió a la curva de enfriamiento del ciclo térmico calculado a través del método $\mathrm{F}(\mathrm{s}, \mathrm{d})$ para placas de acero de $1 / 2$ " de espesor. Estas curvas se caracterizaron a través del parámetro de enfriamiento $\Delta t_{500}^{\mathbf{8 0 0}}$, que indica el tiempo que transcurre mientras se atraviesa un rango de temperaturas de interés, el cual se encuentra comprendido entre $800^{\circ} \mathrm{C}$ y $500^{\circ} \mathrm{C}$ para los aceros de bajo contenido de carbono. En este rango ocurren la mayoría de transformaciones metalúrgicas en el material [13]. 
Desarrollo de un método de simulación física de zonas térmicamente afectadas en soldaduras de acero, para estudios de propagación de grietas por fatiga

La Tabla 3 resume las características de los ciclos térmicos empleados en la simulación de las ZTA que fueron objeto de estudio; estos ciclos permitieron obtener la mejor similitud posible entre las microestructuras simuladas y las contrapartes reales. Se distinguen dos aspectos importantes: en primer lugar, el aporte de calor utilizado en las simulaciones fue diferente al valor reportado en el procedimiento de soldadura respectivo (Tabla 2); el valor reportado corresponde al valor promedio de los diferentes parámetros del proceso de soldadura, y no refleja la variabilidad que se pudo haber presentado al pasar por el punto sobre el cual se analizó la microestructura de la unión soldada. En segundo lugar, la simulación de las zonas de grano grueso requirió del empleo de curvas de enfriamiento obtenidas con un aporte de calor superior al utilizado para simular las zonas de grano fino. Aun cuando ambas zonas fueron producidas por el mismo cordón en la unión soldada, este comportamiento fue consecuencia de la reducida velocidad de calentamiento utilizada en las simulaciones físicas, que en el caso de la zona de grano grueso produjo un tamaño de grano austenítico ligeramente superior al esperado. De esta forma, el porcentaje de ferrita total obtenido se tuvo que compensar aumentando el valor del parámetro $\Delta t_{500}^{800}$.

\section{TABLA 3}

Ciclos tÉRMiCOS UTILIZADOS EN LA SIMULACIÓN DE LAS DIFERENTES ZTA OBJETO DE ESTUDIO. LA

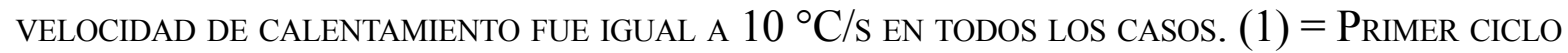
TÉRMICO, (2) = SEGUNDO CICLO TÉRMICO.

\begin{tabular}{|c|c|c|c|c|}
\hline ZTA a simular & Acero & $\mathbf{T}_{\mathbf{m a x}}\left({ }^{\circ} \mathbf{C}\right)$ & $\Delta \boldsymbol{t}_{\mathbf{5 0 0}}^{\mathbf{8 0 0}} \mathbf{( s )}$ & $\begin{array}{c}\text { Aporte de } \\
\text { calor (kJ/cm) }\end{array}$ \\
\hline Grano fino & A283 Gr. C & 901 & 6,54 & 11,00 \\
Grano grueso & A283 Gr. C & 1200 & 11,24 & 13,63 \\
Multipase (1) & A283 Gr. C & 1200 & 14,76 & 14,80 \\
Multipase (2) & A283 Gr. C & 900 & 8,49 & 12,20 \\
\hline Grano fino & A106 Gr. B & 901 & 10,64 & 13,50 \\
Grano grueso & A106 Gr. B & 1200 & 12,67 & 14,20 \\
Multipase (1) & A106 Gr. B & 1200 & 13,20 & 14,30 \\
Multipase (2) & A106 Gr. B & 901 & 10,64 & 13,50 \\
\hline
\end{tabular}

A manera de demostración, la Fig. 8 muestra una comparación entre el ciclo térmico calculado a través del método $\mathrm{F}(\mathrm{s}, \mathrm{d})$ para simular la zona de grano grueso de la unión soldada de acero A106 Gr. B y el ciclo térmico seguido por una de las probetas durante la simulación física respectiva. La única diferencia apreciable entre las curvas está relacionada con las velocidades de calentamiento, como se ha manifestado anteriormente. Se puede observar también que las curvas de temperatura obtenidas en los tres puntos de medición fueron muy similares, lo que explica la homogeneidad de la microestructura a lo largo de la trayectoria de la grieta. Los valores del parámetro $\Delta \boldsymbol{t}_{\mathbf{5 0 0}}^{\mathbf{8 0 0}}$ obtenidos para esta prueba fueron $12,54 \mathrm{~s}$ (TC1, termocupla de control), 13,93 s (TC2) y 12,81 s (TC3), muy cercanos al valor predicho por el ciclo térmico correspondiente (Tabla 3). La Fig. 9 permite comparar solamente las curvas de enfriamiento obtenidas en esta prueba. 

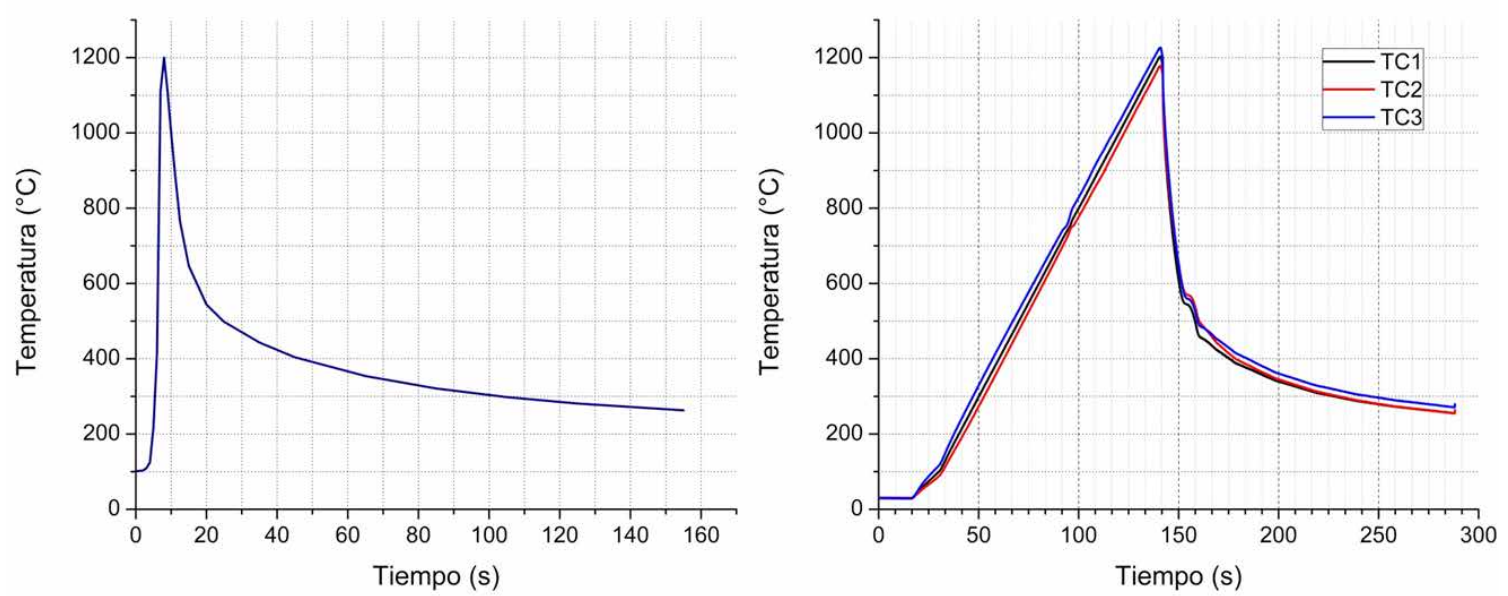

Fig. 8. Comparativa del ciclo térmico calculado a partir del método $\mathrm{F}(\mathrm{s}, \mathrm{d})$ (gráfica izquierda) y el ciclo térmico monitoreado en la simulación de la zona de grano grueso de la unión soldada de acero A106 Gr. B (probeta A106FC3, gráfica derecha).

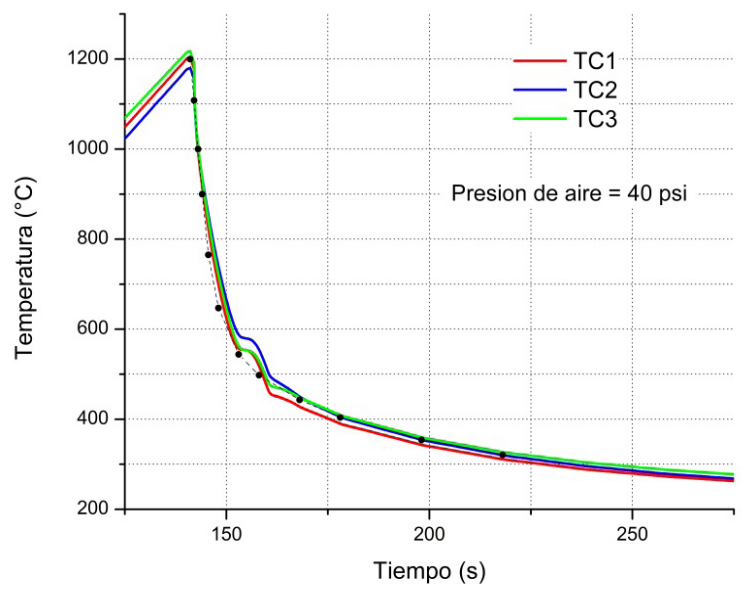

Fig. 9. Curvas de enfriamiento obtenidas en los diferentes puntos de monitoreo de temperatura durante la simulación física de la zona de grano grueso de la unión soldada de acero A106 Gr. B (probeta A106-FC3) por medio del método desarrollado, en comparación con la curva de enfriamiento teórica calculada a través del método $\mathrm{F}(\mathrm{s}, \mathrm{d})$ (puntos negros).

\section{Comparación entre las microestructuras simuladas y las reales}

En primer lugar, la microestructura de los metales base estuvo compuesta, principalmente, por ferrita y perlita; para el acero A283 Gr. C se hizo evidente una estructura de laminación producto del proceso de conformado.
La Tabla 4 muestra los resultados del análisis cuantitativo llevado a cabo en las microestructuras simuladas de la unión soldada de acero A283 Gr. C y sus respectivas contrapartes reales. 
Desarrollo de un método de simulación física de zonas térmicamente afectadas en soldaduras de acero, para estudios de propagación de grietas por fatiga

\section{TABLA 4}

RESULTADOS DEL ANÁLISIS CUANTITATIVO DE LA MICROESTRUCTURA DE LAS ZONAS DE ESTUDIO SIMULADAS Y SUS CONTRAPARTES REALES PARA LA UNIÓN SOLDADA DE ACERO A283 GR. C

\begin{tabular}{|l|c|c|c|c|}
\hline \multirow{2}{*}{ Zona } & \multicolumn{2}{|c|}{ Tamaño de Grano ASTM } & \multicolumn{2}{c|}{ Porcentaje de Ferrita Total } \\
\cline { 2 - 5 } & Real & Simulado & Real & Simulado \\
\hline Grano Fino & $13,1+/-0,3$ & $13,0+/-0,2$ & $70,1 \%+/-0,9 \%$ & $72,6 \%+/-1,1 \%$ \\
\hline Grano Grueso & - & - & $17,6 \%+/-2,1 \%$ & $18,4 \%+/-5,3 \%$ \\
\hline Multipase & $13,0+/-0,3$ & $12,9+/-0,2$ & $78,3 \%+/-2,9 \%$ & $78,3 \%+/-0,3 \%$ \\
\hline Metal Base & $10,7+/-0,2$ & - & $81,9 \%+/-1,5 \%$ & - \\
\hline
\end{tabular}

Como se observa en las Figuras 10 y 11, las microestructuras de la zona de grano fino y de la zona de múltiples pasadas de la unión soldada de acero A283 Gr. C, así como la de las zonas simuladas, estuvieron conformada por ferrita y perlita; las colonias de perlita estuvieron distribuidas de manera más uniforme y fueron más finas en la zona de múltiples pasadas que en la zona de grano fino, lo cual se debe a que la primera fue sometida a un tratamiento térmico adicional de alta temperatura (correspondiente al primer ciclo térmico aplicado). Adicionalmente, la cementita que compone esas colonias de perlita tuvo siempre una morfología laminar, a diferencia de la morfología combinada (laminas irregulares y formas esféricas) que apareció en la zona de grano fino, debido a la velocidad de enfriamiento mayor a la cual estuvo sometida esta última zona. Esto explica también el menor contenido de ferrita en la microestructura. a)

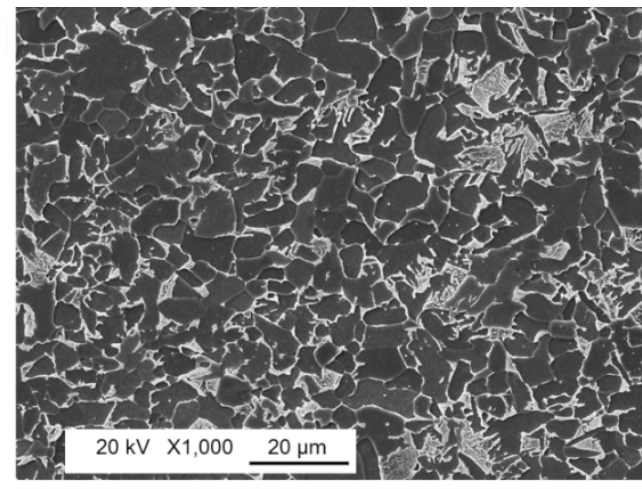

c)

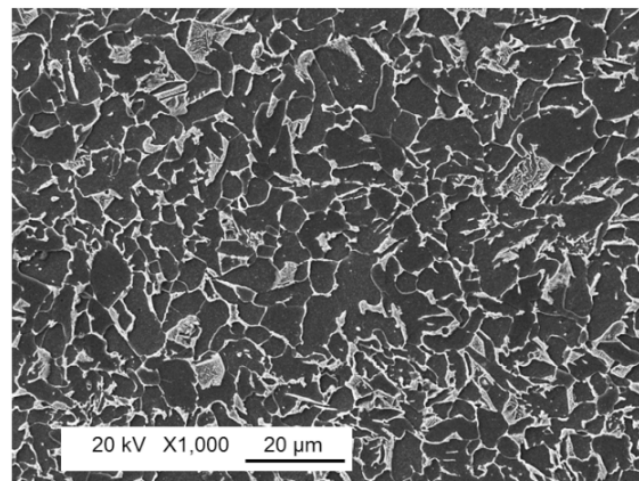

b)

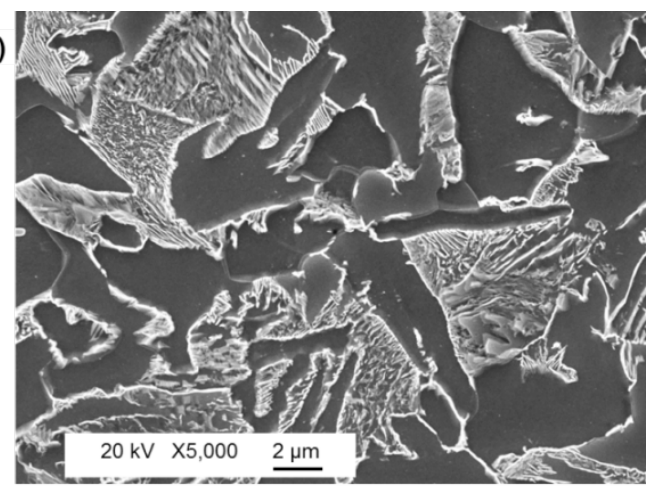

d)

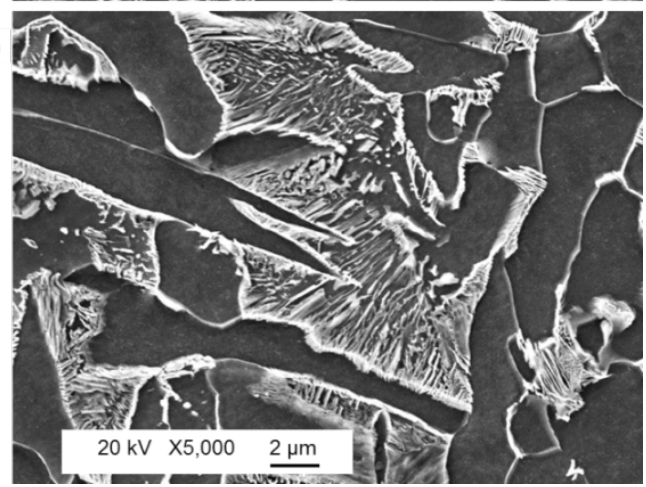

Fig. 10. Microestructura de la zona de grano fino real de la unión soldada de acero A283 Gr. C (a y b) y de la respectiva simulación física (c y d). MEB. 
a)

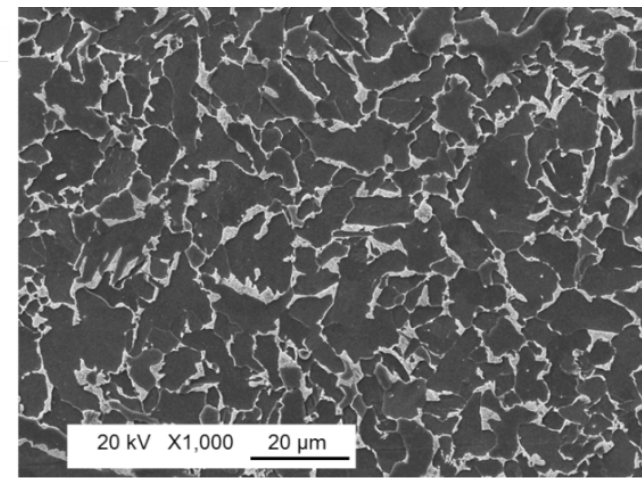

c)

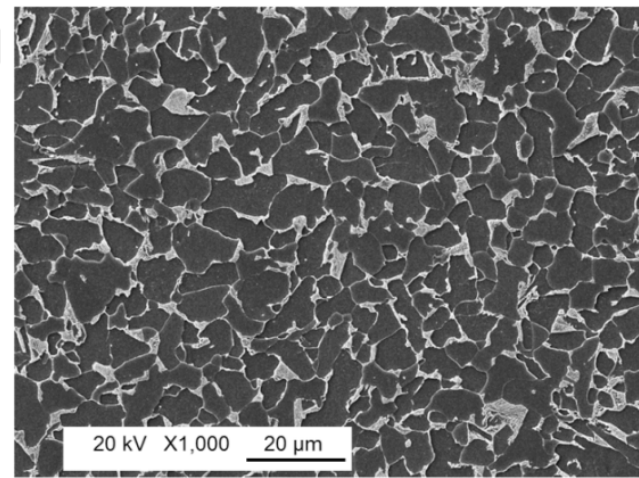

b)

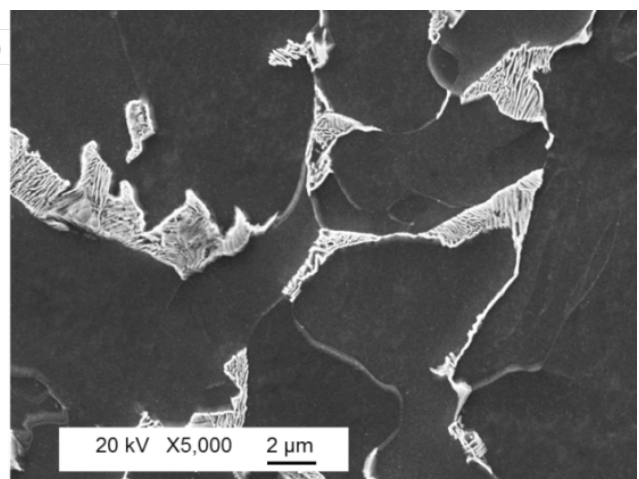

d)

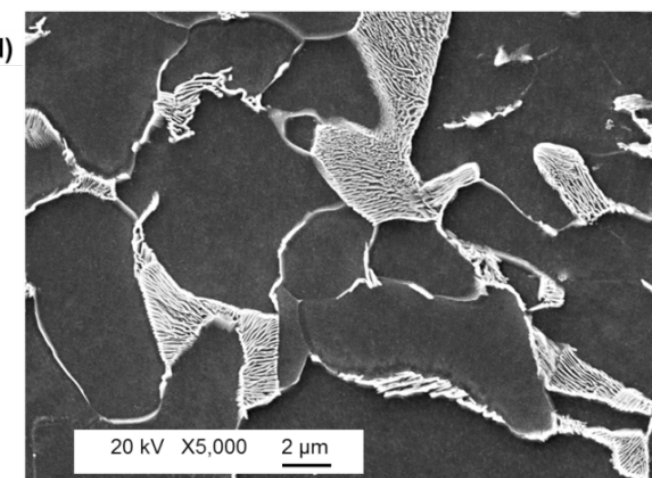

Fig. 11. Microestructura de la zona de múltiples pasadas real de la unión soldada de acero A283 Gr. C (a y b) y de la respectiva simulación física (c y d). MEB.

Por su parte, la microestructura de la zona de grano grueso (Fig. 12) se caracterizó por la presencia muy reducida de ferrita, en comparación con las demás zonas. Esta fase, de apariencia clara en las micrografías por MO y oscura en las micrografías por MEB, estuvo conformada por ferrita poligonal (equiaxial) en pequeñas cantidades, ferrita widmanstatten y ferrita proeuctectoide. El resto de la microestructura se compuso de precipitados finos de carburo de hierro dispersos dentro de granos gruesos de matriz ferrítica, lo que se puede catalogar como un agregado ferritacarburo de hierro. La morfología de los carburos de hierro fue predominantemente esférica, aunque también se presentaron láminas irregulares orientadas en diferentes direcciones. La alta temperatura alcanzada por esta zona del material produjo un crecimiento considerable del grano austenítico que, combinado con la ausencia de compuestos intermetálicos en la microestructura, redujo significativamente el número de sitios de nucleación de ferrita; de esta forma, durante el enfriamiento relativamente rápido se pudo nuclear ferrita a temperaturas intermedias cuando el grado de sobreenfriamiento fue importante. Por la falta de movilidad de los átomos a esas temperaturas, la gran cantidad de austenita inestable se transformó luego en ferrita, precipitando el exceso de carbono localmente, formando el agregado descrito anteriormente. 
Desarrollo de un método de simulación física de zonas térmicamente afectadas en soldaduras de acero, para estudios de propagación de grietas por fatiga

a)

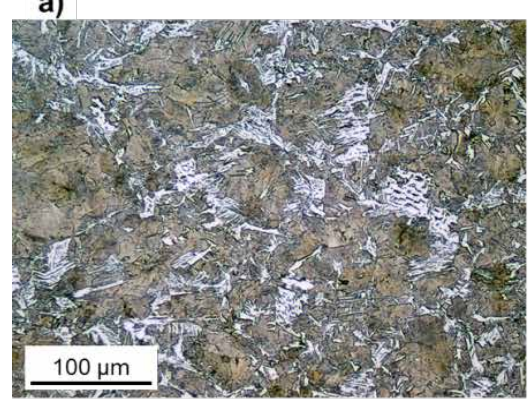

d)

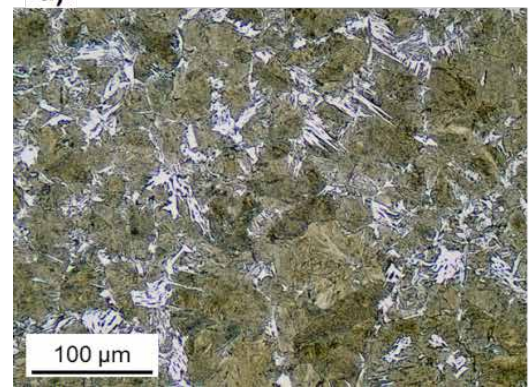

b)

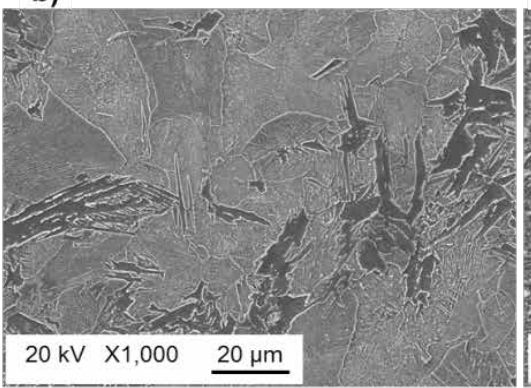

e)

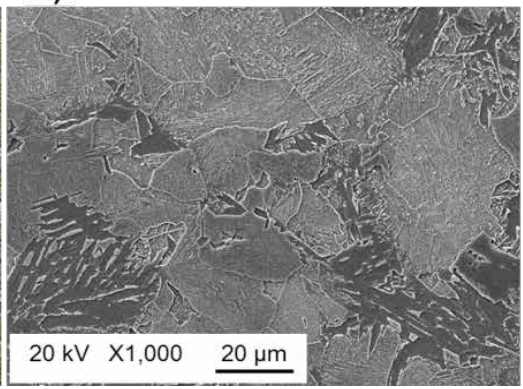

c)

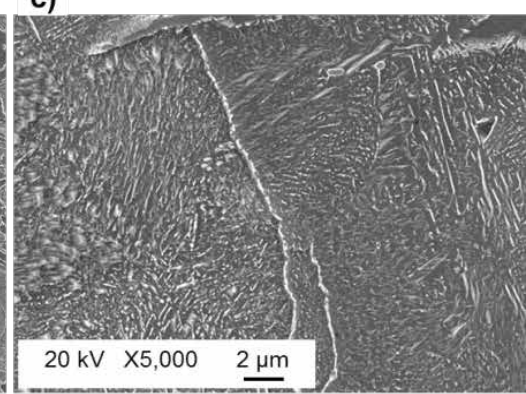

f)

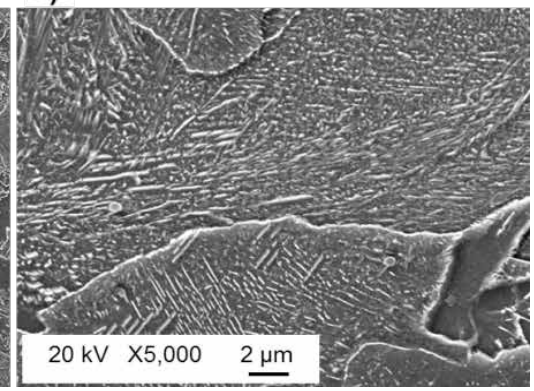

Fig. 12. Microestructura de la zona de grano grueso real de la unión soldada de acero A283 Gr. C (a, b y c) y de la respectiva simulación física (d, e y f). Micrografías a y d: MO. Micrografías b, c, e y f: MEB.

Por otro lado, la Tabla 5 muestra los resultados del análisis cuantitativo llevado a cabo en las microestructuras simuladas de la unión soldada de acero A106 Gr. B y sus respectivas contrapartes reales.

\section{TABLA 5}

RESULTADOS DEL ANÁLISIS CUANTITATIVO DE LA MICROESTRUCTURA DE LAS ZONAS DE ESTUDIO SIMULADAS Y SUS CONTRAPARTES REALES PARA LA UNIÓN SOLDADA DE ACERO A106 GR. B

\begin{tabular}{|l|c|c|c|c|}
\hline \multirow{2}{*}{ Zona } & \multicolumn{2}{|c|}{ Tamaño de Grano ASTM } & \multicolumn{2}{c|}{ Porcentaje de Ferrita Total } \\
\cline { 2 - 5 } & Real & Simulado & Real & Simulado \\
\hline Grano Fino & $12,3+/-0,1$ & $12,3+/-0,3$ & $70,7 \%+/-0,6 \%$ & $68,6 \%+/-1,6 \%$ \\
\hline Grano Grueso & - & - & $43,4 \%+/-1,4 \%$ & $40,0 \%+/-4,2 \%$ \\
\hline Multipase & $12,7+/-0,2$ & $12,7+/-0,2$ & $76,3 \%+/ 1,3 \%$ & $78,0 \%+/-1,2 \%$ \\
\hline Metal Base & $8,6+/-0,2$ & - & $78,6 \%+/-1,4 \%$ & - \\
\hline
\end{tabular}

En este caso, las zonas de grano fino (Fig. 13) y las zonas de múltiples pasadas reales (Fig. 14) mostraron un comportamiento muy similar al descrito para el acero A283 Gr. C. No obstante, los tamaños de grano
ASTM obtenidos fueron inferiores debido al tamaño de grano ASTM del metal base, que también fue inferior. Aun así, la simulación física permitió reproducir este comportamiento con un alto grado de exactitud. 
a)

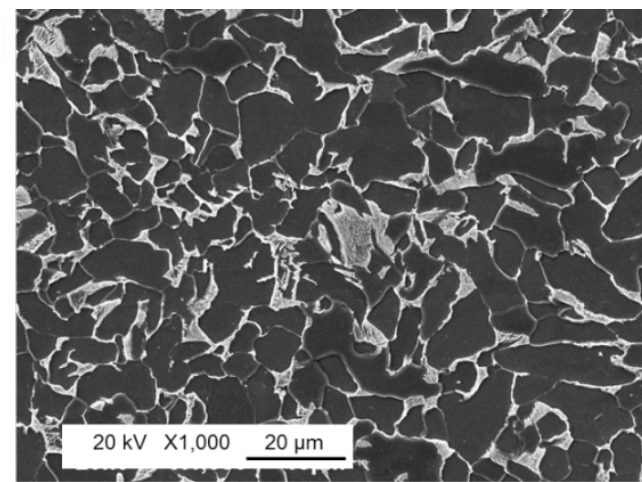

c)

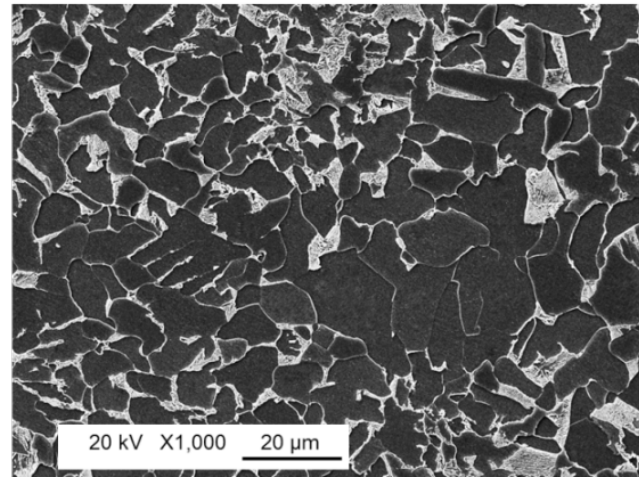

b)

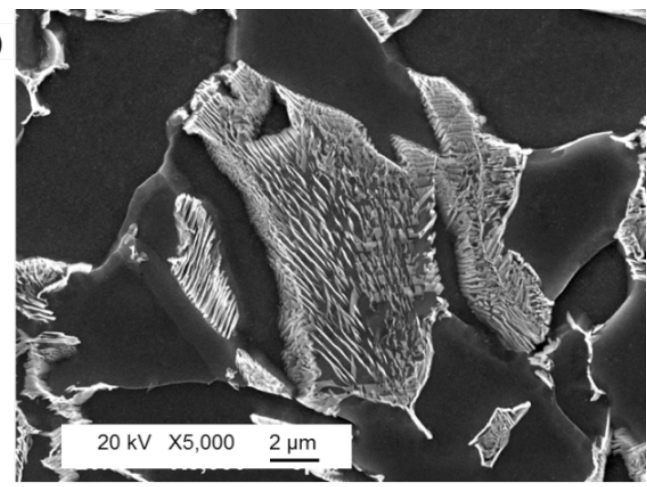

d)

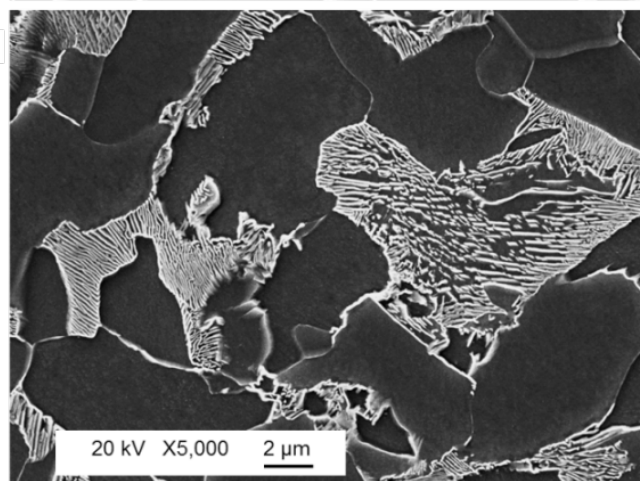

Fig. 13. Microestructura de la zona de grano fino real de la unión soldada de acero A106 Gr. B (a y b) y de la respectiva simulación física (c y d). MEB.

Las velocidades de enfriamiento en la junta del acero A106 Gr. B fueron más lentas, lo que en general permitió mayores tiempos para la difusión y reacomodamiento de los átomos durante la transformación alotrópica; esto posibilitó que, por ejemplo, la zona de grano grueso (Fig. 15) tuviera un contenido de ferrita total mayor que en el caso de la unión soldada de acero A283 Gr. C. Este contenido estuvo compuesto por ferrita proeuctetoide y ferrita widmanstatten, principalmente, $\mathrm{y}$, en menor medida, por ferrita poligonal y ferrita acicular; el resto de la microestructura correspondió al agregado ferritacarburo de hierro, aunque en este caso la morfología de los precipitados se asemejó más a láminas irregulares que a formas esféricas, debido, precisamente, a las velocidades de enfriamiento menores. 
Desarrollo de un método de simulación física de zonas térmicamente afectadas en soldaduras de acero, para estudios de propagación de grietas por fatiga

a)

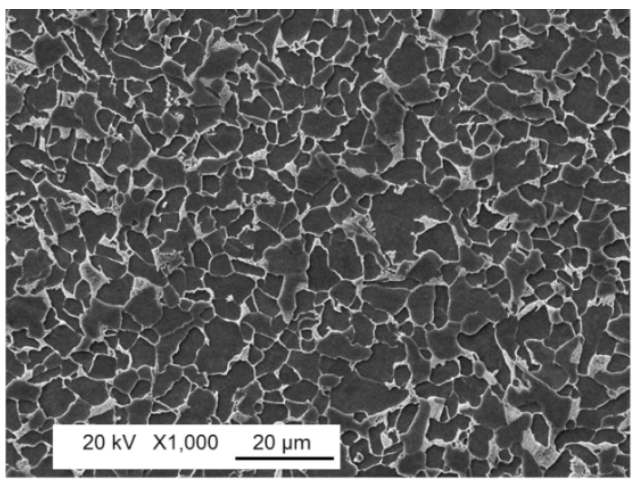

c)

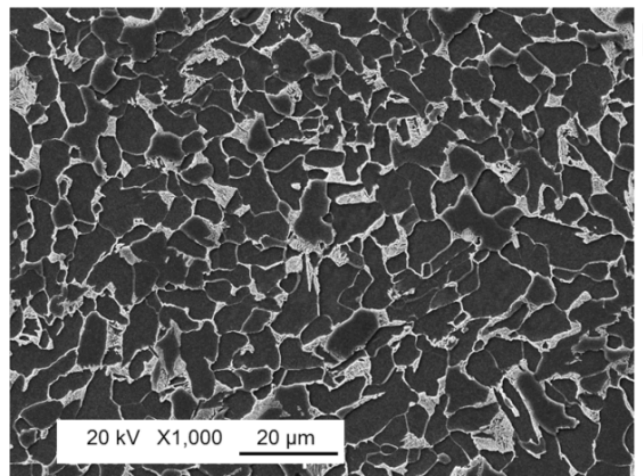

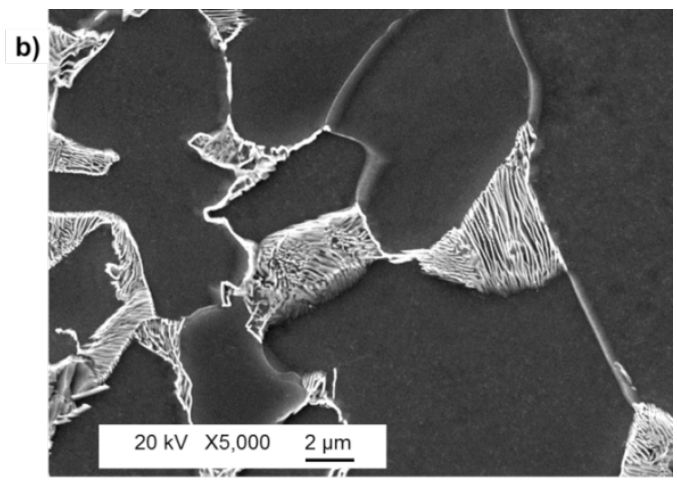

d)

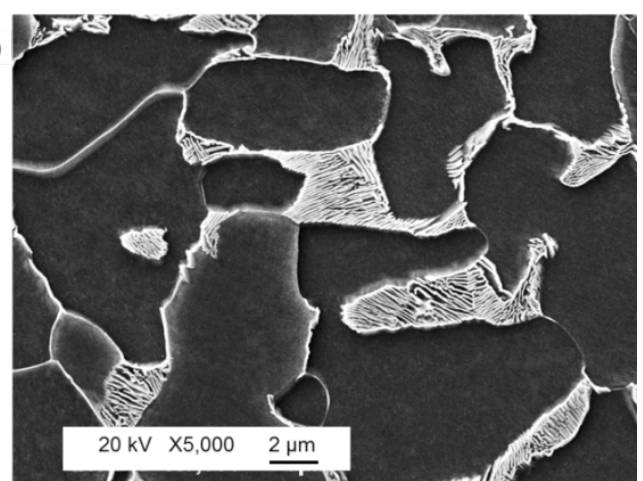

Fig. 14. Microestructura de la zona de múltiples pasadas real de la unión soldada de acero A106 Gr. B (a y b) y de la respectiva simulación física (c y d). MEB.

a)

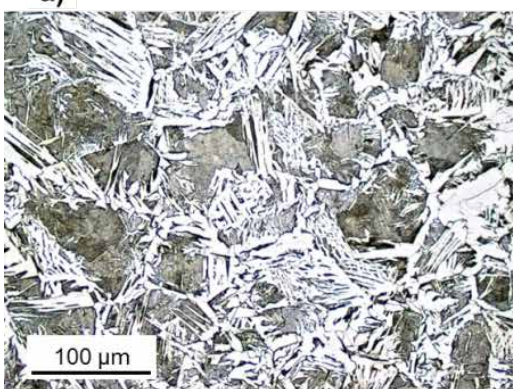

d)

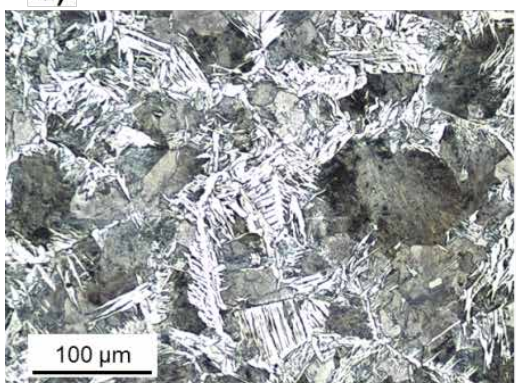

b)

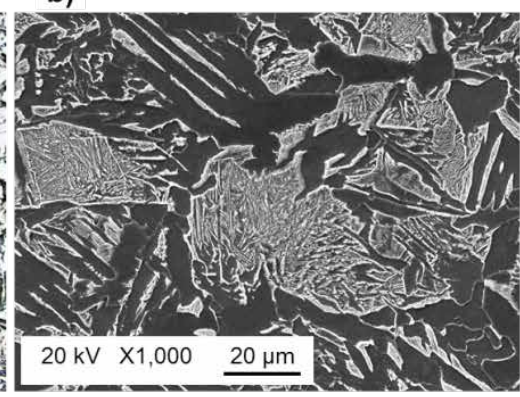

e)

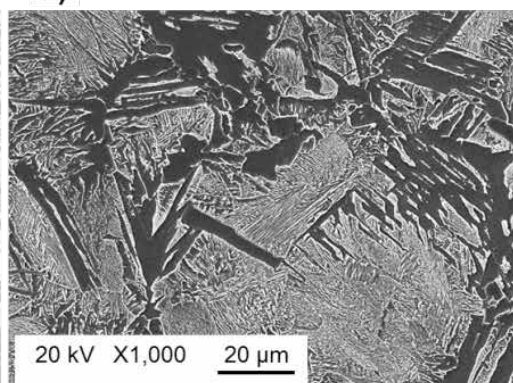

c)

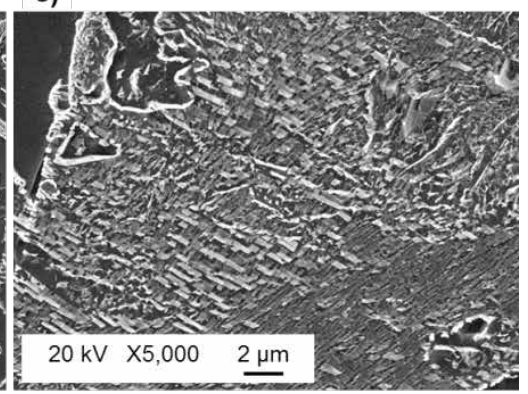

f)

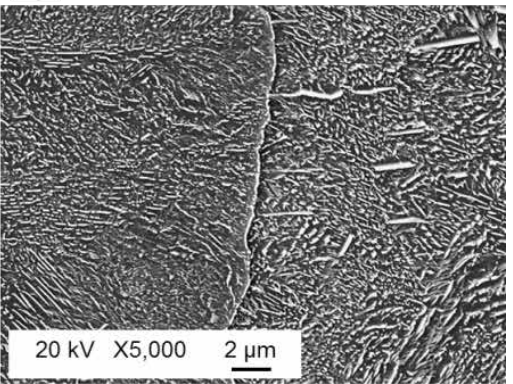

Fig. 15. Microestructura de la zona de grano grueso real de la unión soldada de acero A106 Gr. B (a, b y c) y de la respectiva simulación física (d, e y f). Micrografías a y d: MO. Micrografías b, c, e y f: MEB. 
Deestemodo, fueposiblereproducirsatisfactoriamente, con un alto grado de similitud, las características microestructurales presentes en las ZTA de interés de las uniones soldadas de los aceros A283 Gr. C y A106 Gr. B fabricadas a través del proceso FCAW, en una región considerablemente grande del material base correspondiente.

\section{Propiedades mecánicas resultantes}

Las Tablas 6 y 7 resumen los resultados de las pruebas de tensión llevadas a cabo en las probetas simuladas y en el metal base de los aceros A283 Gr. C y A106 Gr. $\mathrm{B}$, respectivamente. Es de destacar la imposibilidad de llevar a cabo este tipo de pruebas sobre la ZTA real de la unión soldada, debido al tamaño reducido que presentan estas zonas, lo que impide mecanizar las probetas necesarias. En general, la resistencia aumenta cuando se incrementa el tamaño de grano ASTM y se disminuye el contenido de ferrita total en la microestructura (la fase menos resistente). Como era de esperar, lo contrario sucede con la ductilidad. Las propiedades mecánicas de las zonas de grano grueso, que por lo general concentran los problemas de origen metalúrgico que se producen en la ZTA, revelan que son las más propensas a la falla dentro de las uniones soldadas estudiadas, pues combinan las mayores durezas con las resistencias más altas y las más bajas ductilidades; sin embargo, este comportamiento no es muy alarmante, ya que la combinación de propiedades por sí solas no lleva a un descenso muy significativo del desempeño mecánico de la junta.

\section{TABLA 6}

RESULTADOS DE ENSAYOS MECÁNICOS EN LAS PROBETAS SIMULADAS DE LA ZTA PARA LA UNIÓN

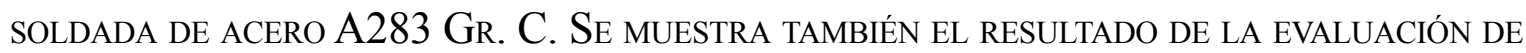
MICRODUREZA EN LAS RESPECTIVAS CONTRAPARTES REALES Y LOS RESULTADOS MEDIDOS EN EL METAL BASE.

\begin{tabular}{|l|c|c|c|c|c|}
\hline \multirow{2}{*}{ Zona } & \multirow{2}{*}{$\begin{array}{c}\text { Resistencia a la } \\
\text { cedencia (MPa) }\end{array}$} & $\begin{array}{c}\text { Resistencia } \\
\text { máxima (MPa) }\end{array}$ & \multirow{2}{*}{ Ductilidad } & \multicolumn{2}{c|}{ Dureza (HV) } \\
\cline { 3 - 6 } & $488 \pm 23$ & $508 \pm 1$ & $30,56 \% \pm 1,30 \%$ & $179,7 \pm 7,6$ & $167,4 \pm 0,3$ \\
\hline Grano Fino & $374 \pm 13$ & $552 \pm 3$ & $26,02 \% \pm 4,93 \%$ & $207,4 \pm 13,7$ & $181,0 \pm 1,8$ \\
\hline Grano Grueso & $377 \pm 18$ & $520 \pm 7$ & $34,30 \% \pm 4,75 \%$ & $179,6 \pm 3,3$ & $157,2 \pm 2,7$ \\
\hline Multipase & $340 \pm 36$ & $464 \pm 10$ & $41,02 \% \pm 1,91 \%$ & $170,1 \pm 7,3\left(^{*}\right)$ & - \\
\hline Metal Base & & &
\end{tabular}

$\left(^{*}\right)$ Microdureza del metal base medida en la unión soldada. Microdureza real del metal base es 139,8 HV ( $\left.\pm 7,3\right)$

\section{TABLA 7}

RESULTADOS DE ENSAYOS MECÁNICOS EN LAS PROBETAS SIMULADAS DE LA ZTA PARA LA UNIÓN SOLDADA DE ACERO A106 Gr. B. SE MUESTRA TAMBIÉN El RESUlTAdo DE LA EVALUACiÓN DE MICRODUREZA EN LAS RESPECTIVAS CONTRAPARTES REALES Y EN EL METAL BASE

\begin{tabular}{|l|c|c|c|c|c|}
\hline \multirow{2}{*}{ Zona } & \multirow{2}{*}{$\begin{array}{c}\text { Resistencia a la } \\
\text { cedencia (MPa) }\end{array}$} & $\begin{array}{c}\text { Resistencia } \\
\text { máxima (MPa) }\end{array}$ & \multirow{2}{*}{ Ductilidad } & \multicolumn{2}{c|}{ Dureza (HV) } \\
\cline { 3 - 6 } & $439 \pm 32$ & $558 \pm 26$ & $34,31 \% \pm 0,07 \%$ & $198,3 \pm 8,2$ & $174,2 \pm 3,9$ \\
\hline Grano Fino & $443 \pm 17$ & $621 \pm 14$ & $23,66 \% \pm 1,23 \%$ & $214,0 \pm 10,9$ & $196,6 \pm 3,9$ \\
\hline Grano Grueso & $466 \pm 30$ & $561 \pm 9$ & $34,82 \% \pm 1,88 \%$ & $169,5 \pm 4,2$ & $174,4 \pm 0,1$ \\
\hline Multipase & $499 \pm 29$ & $584 \pm 3$ & $29,60 \% \pm 1,78 \%$ & $172,6 \pm 12,2\left(^{*}\right)$ & - \\
\hline Metal Base & & &
\end{tabular}

$\left({ }^{*}\right)$ Microdureza del metal base medida en la unión soldada. Microdureza real del metal base es 166,3 HV ( $\left.\pm 9,8\right)$ 
Lo que sí puede ser preocupante es el nivel de esfuerzos residuales que se originan en la unión soldada, a pesar de que los procedimientos de soldadura hayan sido diseñados, construidos y evaluados de acuerdo con los códigos de construcción soldada; esto se refleja en las mediciones de microdureza tan diferentes que se obtuvieron entre una zona simulada y su respectiva contraparte real, a pesar de que ambas zonas presentaran prácticamente las mismas características microestructurales. El valor de microdureza difiere un máximo de $26 \mathrm{HV}$ (para la zona de grano grueso de la unión soldada de acero A283 Gr. C). De hecho, el valor de microdureza medido en el metal base del acero A283 Gr. C se reduce también, aproximadamente, 30 HV cuando se mide en cupones extraídos de la misma fuente y no han sido utilizados para fabricar la unión soldada. La dureza, al ser una medida de la resistencia del metal a la deformación plástica permanente [14], resulta afectada por los esfuerzos residuales en la medida que estos se suman a los esfuerzos inducidos en el material al realizar la indentación en la determinación de la dureza. Por lo tanto, la huella puede tener un tamaño diferente en función de la magnitud y el sentido de los esfuerzos residuales. De esta manera, los valores de microdureza obtenidos directamente sobre la unión soldada no son consecuencia única de las características microestructurales. Asimismo, la dispersión de las mediciones de microdureza tomadas sobre las probetas simuladas es sensiblemente menor, lo que muestra que la distribución de los esfuerzos residuales en la junta es irregular; esto representa un riesgo potencial muy importante para las uniones soldadas si ahora se tiene en cuenta la combinación de propiedades mecánicas mencionada anteriormente y su cercanía a los concentradores de esfuerzo (por ejemplo, el pie de la soldadura).
Los hechos anteriores, sumados a los defectos que normalmente se originan en la junta durante un procedimiento de soldadura, representan una prueba clara de la inconveniencia de utilizar probetas soldadas para estudiar el efecto neto de la microestructura sobre la propagación de grietas por fatiga en estos elementos. Al contrario, las ventajas de llevar a cabo estos estudios con probetas simuladas físicamente a través del sistema Gleeble son evidentes, en la medida que se puede estudiar el efecto neto de la microestructura variable de la ZTA en la propagación de grietas por fatiga, puesto que se pudieron reproducir las características microestructurales en secciones del metal base suficientemente grandes como para llevar a cabo controladamente este tipo de pruebas, en ausencia de otros factores que pueden influenciar los resultados (como por ejemplo, los esfuerzos residuales).

Finalmente, y a manera de ilustración, la Fig. 16 muestra algunos resultados del ensayo de propagación de grietas por fatiga realizado con las probetas simuladas. Se observan comportamientos diferentes en función de la microestructura ensayada. En particular, se observa que la zona de grano grueso de la unión soldada de acero A106 Gr. B presenta las velocidades de crecimiento de grieta (da/ dN) más bajas para determinado rango del factor concentrador de esfuerzos $(\Delta K)$; esto quiere decir que el comportamiento bajo el régimen de carga estática es contrario al comportamiento bajo condiciones dinámicas de propagación de grieta en esta zona, probablemente ayudado por el contenido de ferrita acicular presente en la microestructura referida. De este modo, es posible establecer que la microestructura de la ZTA sí afecta la velocidad de propagación de grietas por fatiga en las uniones soldadas de aceros, al contrario de lo que se ha afirmado hasta ahora en la literatura. 


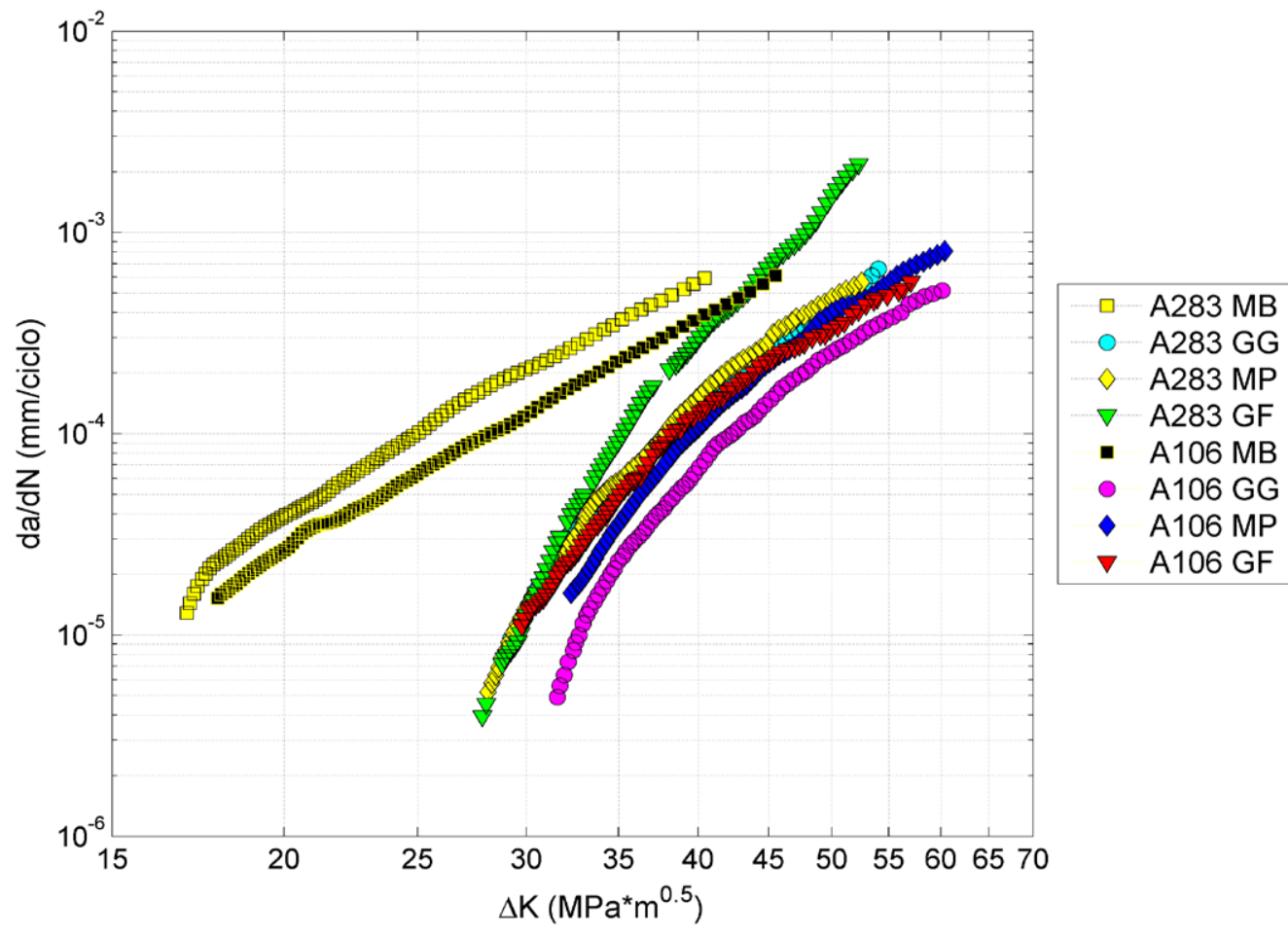

Fig. 16. Resultados preliminares de los ensayos de propagación de grietas por fatiga realizados con probetas de la ZTA simuladas. Se incluyen también resultados de los metales base.

\section{Conclusiones}

El método desarrollado en el presente trabajo para simular físicamente, a través del sistema Gleeble, las ZTA de las uniones soldadas de aceros estructurales fabricadas a través del proceso FCAW permitió reproducir las características microestructurales de las zonas de interés en secciones significativamente grandes del metal base correspondiente de manera separada. Las microestructuras simuladas, libres de defectos y esfuerzos residuales, presentaron características muy similares a su contraparte real, a pesar de las enormes dificultades que supone el empleo de probetas que son considerablemente más grandes que las utilizadas normalmente en las simulaciones. Lo anterior permitió llevar a cabo ensayos de tensión y propagación de grietas por fatiga para estudiar el efecto de la variación microestructural de la ZTA sobre el comportamiento mecánico de la junta, especialmente bajo condiciones de fatiga. Asimismo, se establecieron las características del ciclo térmico que produjo cada zona objeto de estudio, y se pudo comprobar que la microestructura afecta la velocidad de propagación de grietas por fatiga.

\section{Agradecimientos}

Los autores agradecen a la empresa Controltec Ltda., a la Universidad del Valle, a Colciencias y al Instituto Madrileño de Estudios Avanzados de Materiales, Instituto IMDEA Materiales, por el apoyo fundamental ofrecido durante el desarrollo del trabajo.

\section{REFERENCIAS}

[1] J. Barsom and S. Rolfe, Fracture and fatigue control in structures: applications of fracture mechanics. United Kingdom: ButterworthHeinemann, 1999.

[2] D. F. Atehortua-López, "Propagación de grietas por fatiga en uniones soldadas por FCAW de aceros de bajo carbono y aceros de baja aleación y la aplicabilidad del ultrasonido como herramienta de monitoreo en este tipo 
de estudios". Tesis de doctorado, Facultad de Ingeniería, Universidad del Valle, Cali, Colombia, 2016.

[3] T. Lassen and N. Recho, Fatigue Life Analyses of Welded Structures: Flaws. London: WileyISTE, 2006.

[4] ASM International Handbook Committee, ASM Handbook: Fatigue and Fracture, vol. 19, ASM International, 1996.

[5] Y. Adonyi, "Heat-Affected Zone Characterization by Physical Simulations", Welding Journal, vol. 85 (10), pp. 42-47, Oct. 2006.

[6] J. A. Gianetto, F. Fazeli, Y. Chen et al., "Microstructure and Toughness of Simulated Grain Coarsened Heat Affected Zones in X80 Pipe Steels", 10th International Pipeline Conference, Calgary, Canada, 2014.

[7] A. Polyakov, D. Gunderov, V. Sitdikov et al., "Physical Simulation of Hot Rolling of Ultrafine Grained Pure Titanium", Metallurgical and Materials Transactions B, vol. 45 (6), pp. 2315-2326, Dec. 2014. DOI: http://dx.doi. org/10.1007/s11663-014-0133-9.

[8] M. Rahimian, S. Milenkovic, L. Maestro et al., "Physical Simulation of Investment Casting of Complex Shape Parts", Metallurgical and
Materials Transactions A, vol. 46 (5), pp. 22272237, May. 2015.

[9] Z. Gao and J. Niu, "Study on microstructure and impact ductility of simulated weld HAZ of high-strength wear-resistant steel NM360", Reviews on advanced materials science, vol. 33 (3), pp. 232-237, Nov. 2013.

[10] B. Liu, J. Qu and W. Sun, "Effects of thermal cycle on mechanical properties and fractography in HAZ of HQ130 steel", Acta Metallurgica Sinica, vol. 17 (3), pp. 274-278, Jun. 2004.

[11] C. Zhou and C. D. Lundin, "A Comparison of Published Haz Thermal Simulation Methods Used to Derive Weld Haz Thermal Cycles", Acta Metallurgica Sinica, vol. 13 (1), pp. 223232, Feb. 2000.

[12] G. Atkins, D. Thiessen, N. Nissley and Y. Adonyi, "Welding Process Effects in Weldability Testing of Steels", Welding Journal, vol. 81 (4), pp. 61s-68s, Apr. 2002.

[13] R. Blondeau, Metallurgy and Mechanics of Welding: Processes and Industrial Applications. London: ISTE Ltd. and John Wiley \& Sons, Inc., 2008.

[14] G. E. Dieter, Mechanical Metallurgy: SI Metric Edition. Boston: McGraw-Hill, 1988. 